\title{
Pacific
}

Journal of

Mathematics

\section{THE CAUCHY INTEGRAL, ANALYTIC CAPACITY AND SUBSETS OF QUASICIRCLES}

\author{
XIANG FANG
}




\section{THE CAUCHY INTEGRAL, ANALYTIC CAPACITY AND SUBSETS OF QUASICIRCLES}

\section{XIANG FANG}

In this paper, we show that if $K \subset \mathbb{C}$ is $A D$-regular and sufficiently flat, then $K$ is a subset of a chord-arc curve if the Cauchy integral operator is bounded on $L^{2}(K)$. This result partially answers a question raised by $G$. David, P. Jones and S. Semmes. Also, we prove that if $K$ is as above (locally) and has positive analytic capacity, then $K$ must contain a subset of a rectifiable curve of positive length. Finally, we characterize subsets of some quasicircles in terms of a simple geometric condition invented by P. Jones.

Introduction. Let $K \subset \mathbb{C}$ be a bounded set and, for $\delta>0$, write

$$
\Lambda^{\delta}(K)=\inf \left\{\sum_{j=1}^{\infty} \delta_{j}: K \subset \bigcup_{j=1}^{\infty} D\left(a_{j}, \delta_{j}\right) ; \delta_{j} \leq \delta\right\}
$$

where $D\left(a_{j}, \delta_{j}\right)=\left\{z:\left|z-a_{j}\right| \leq \delta_{j}\right\}$. Then $\Lambda^{\delta}(K)$ is a decreasing function of $\delta$. The one-dimensional Hausdorff measure $\Lambda(\cdot)$ is defined by

$$
\Lambda(K)=\lim _{\delta \rightarrow 0} \Lambda^{\delta}(K) .
$$

If $K$ is connected and $\Lambda(K)<\infty$, then we call it a rectifiable curve.

Definition 1. A $\Lambda$-measurable set $K \subset \mathbb{C}$ is said to be regular in the sense of Ahlfors and David, or AD-regular, if there exists $M<\infty$ such that for all $x \in K$ and $0<r \leq \operatorname{diam}(K)$,

$$
M^{-1} r \leq \Lambda(K \cap D(x, r)) \leq M r .
$$

If $K$ is connected we call it an AD-regular curve. 
A special kind of AD-regular curve $\Gamma$ is a chord-arc curve which is defined by the condition that, for any $z_{1}, z_{2} \in \Gamma, \Lambda\left(\Gamma\left(z_{1}, z_{2}\right)\right) \leq$ $C\left|z_{1}-z_{2}\right|$, where $\Gamma\left(z_{1}, z_{2}\right)$ is the smallest arc between $z_{1}$ and $z_{2}$ and $C$ is a constant independent of $z_{1}$ and $z_{2}$.

Definition 2. A bounded $\Lambda$-measurable set $K \subset \mathbb{C}$ is said to be B-regular, if it is a subset of a countable union of rectifiable curves.

Let $\mathcal{D}$ be the collection of all dyadic squares. The following very essential geometric quantity was introduced by P. Jones [19]: For a square $Q \in \mathcal{D}$,

$$
\beta_{K}(Q)=\inf _{L} \ell(Q)^{-1} \sup _{z \in K \cap 3 Q} \operatorname{dist}(z, L)
$$

where the infimum is taken over all straight lines $L, \ell(Q)$ is the side length of $Q$ and the square $3 Q$ is the square with the same center as $Q$ and $\ell(3 Q)=3 \ell(Q)$. Let

$$
\beta(K)=\sup _{Q \in \mathcal{D}} \beta_{K}(Q) .
$$

Definition 3. Suppose that $K \subset \mathbb{C}$ is compact. Then $K$ is called locally flat if, for any $\left\{Q_{n}\right\} \subset \mathcal{D}$, satisfying $\lim _{n \rightarrow \infty} \ell\left(Q_{n}\right)=0$,

$$
\lim _{n \rightarrow \infty} \beta_{K}\left(Q_{n}\right)=0 \text {. }
$$

Definition 3 means that points of a locally flat set are, locally, around a straight line. Examples of locally flat sets are subsets of smooth curves and some snowflake type sets (see §3).

The analytic capacity of a compact set $K$ is defined by the following:

Definition 4. Assume $\mathrm{K}$ is a compact subset of $\mathbb{C}$. Then

$$
\gamma(K)=\sup \left\{\left|f^{\prime}(\infty)\right|: f \in H^{\infty}(\Omega), f(\infty)=0,\|f\|_{L^{\infty}} \leq 1\right\}
$$

where

$$
f^{\prime}(\infty)=\lim _{z \rightarrow \infty} z f(z)
$$

$\Omega=\mathbb{C} \backslash K$, and $H^{\infty}(\Omega)=\{$ all analytic bounded functions on $\Omega\}$.

It is easy to see $\gamma(K)$ is positive if and only if $H^{\infty}(\Omega)$ has nontrivial functions. Let $\mathcal{C}$ be the Cauchy integral operator. The main results of this paper are: 
TheOREM I. Suppose $K \subset \mathbb{C}$ is $A D$-regular and suppose that $\mathcal{C}$ is bounded on $L^{2}(K)$ and $\beta(K)$ is sufficiently small with respect to the $L^{2}$ norm of $\mathcal{C}$ and the constant of $A D$-regularity. Then $K$ is contained in a chord-arc curve.

THEOREM II. Suppose $K$ is AD-regular and locally flat. Then $\gamma(K)>0$ if and only if there exits a rectifiable curve $\Gamma$ such that $\Lambda(K \cap \Gamma)>0$.

Theorem III. Suppose $K \subset \mathbb{C}$ is compact. Then, if $\beta(K)$ is small enough, $K$ is a subset of a quasicircle with small constant (see $§ 1)$. Conversely, if $K$ is a quasicircle with small constant, then $\beta(K)$ is small.

Theorem III, which was suggested by P. Jones characterizes sets $K$ with small $\beta(K)$. See $\S 1$ Theorem 1.2 for the details of Theorem III. Theorem I, II partially answer the following open problems:

Problem 1. Suppose $K$ is AD-regular and $\mathcal{C}$ is bounded on $L^{2}(K)$. Is it true that $K$ is contained in an AD-regular curve ?

Problem 2. Suppose $\Lambda(K)<\infty$. Does $\gamma(K)=0$ if and only if $K$ is B-irregular?

Equivalently, we may state Problem 2 as follows:

Problem $2^{\prime}$. Suppose $\Lambda(K)<\infty$. Then $\gamma(K)>0$ if and only if there exists a rectifiable curve $\Gamma$ such that $K \cap \Gamma$ has positive one-dimensional Hausdorff measure.

These problems have been raised by G. David, P. Jones and $\mathrm{S}$. Semmes, and they suggest a connection between $L^{2}$-boundedness of $\mathcal{C}$ and geometric properties of sets. In 1980, G. David [10] proved that Problem 1 is true if $K$ is connected. Later on, G. David and $\mathrm{S}$. Semmes $[\mathbf{1 1}]$ proved that Problem 1 is true if we replace $\mathcal{C}$ by all singular integral operators with odd kernels.

Remark. In Problem 1, the AD-regular condition is necessary. Actually, it is not hard to construct a set $K$ which is not contained in any $\mathrm{AD}$ curve, or even any rectifiable curve, but, $\mathcal{C}$ is bounded on $L^{p}(K), 1<p<\infty$.

It has long been known that the Cauchy integral and analytic capacity are intimately related. Calderón's theorem [3] solved what was known as the Denjoy conjecture: 
THEOREM. Suppose $K$ is a subset of a rectifiable curve. Then $\gamma(K)>0$ if and only if $\Lambda(K)>0$.

Another old conjecture is that $\gamma(K)>0$ if and only if $\operatorname{Fav}(K)>$ 0 , where $\operatorname{Fav}(\cdot)$ is Favard length,

$$
\operatorname{Fav}(K)=\int_{0}^{\pi} \Lambda\left(K_{\theta}\right) d \theta
$$

and where $K_{\theta}$ is the orthogonal projection of $K$ onto the line $\mathbb{R} e^{i \theta}$. This is known to be false for sets where $K$ has non $\sigma$-finite $\Lambda$ measure [22].

Besicovitch [2] proved that, for the sets satisfying $0<\Lambda(K)<\infty$, $\operatorname{Fav}(K)=0$ if and only if $\Lambda(K \cap \Gamma)=0$ for every rectifiable curve $\Gamma$. We call these kind of sets B-irregular. This leads to Problem 2. It follows from Calderón's theorem that $\gamma(K)=0$ implies $K$ is B-irregular. So what we need to know is only whether $\gamma(K)=0$ if $K$ is B-irregular. For example, in 1970, Garnett [15] proved the $\frac{1}{4}$-Cantor set, which is $\mathrm{B}$-irregular and $\mathrm{AD}$ regular, has zero analytic capacity. In 1987, Mattila [25] showed that $\gamma(K)=0$ if $K$ is Birregular and satisfies a certain geometric condition, which we call Mattila's condition (see §3). But, we still do not know much more about Problem 2. In $\S 3$, we prove that the set $K$ in Theorem II fails to satisfy Mattila's condition; thus, Theorem II extends the results we known so far.

Notations. We will use the following notation: $\mathbb{R}$ is the real line, $\mathbb{C}$ is the plane. $\arg (z)$ denotes the argument of $z$, the value of $\arg (z)$ is taken modulo $2 \pi$. $\Re(z)$ and $\Im(z)$ are the real and imaginary parts of $z$, respectively, and $[a, b]$ is the straight line segment from $a$ to $b$. If $\Gamma$ is a Jordan curve, and $a, b \in \Gamma$ we let $\Gamma(a, b)$ denote the arc of smaller diameter between $a$ and $b$.

1. Quasiconformal circles with small estimate constant. Recall that $\Gamma$ is called a quasicircle, if $\Gamma=g(\mathbb{R})$ where $g$ is a quasiconformal mapping of $\mathbb{C}$ into $\mathbb{C}$. More geometrically, $\Gamma$ is a quasicircle iff $\Gamma$ satisfies the "three points condition"

$$
\left|z_{1}-z_{2}\right| \leq Q\left|z_{1}-z_{3}\right|
$$


for a constant $Q$ and any three points on $\Gamma$ with $z_{2}$ on the arc of smaller diameter between $z_{1}$ and $z_{3}$ (see [1], Theorem IV. 5).

It is interesting to characterize a set $K$ contained in a quasicircle in terms of the geometric quantity $\beta(K)$. For that purpose we introduce the following:

Definition 1.1. A Jordan curve is called an s-quasicircle (s denoting "small") with constant $\varepsilon$, if it satisfies the following three points condition: for any three points $z_{1}, z_{2}, z_{3}$, on the curve such that $z_{2}$ separates $z_{1}$ and $z_{3}$, we have

$$
\left|z_{1}-z_{2}\right|+\left|z_{2}-z_{3}\right| \leq(1+\varepsilon)\left|z_{1}-z_{3}\right|
$$

It is clear that an s-quasicircle with constant $\varepsilon$ is a quasicircle with constant $1+\varepsilon$ but, its converse is not true. We establish the following result which is a precise version of Theorem III:

THEOREM 1.2. Suppose $K \subset \mathbb{C}$ is compact and suppose $\varepsilon<\frac{1}{100}$. Then $K$ is the subset of an s-quasicircle with constant $C_{0} \varepsilon$, if it satisfies

$$
\beta(K) \leq \delta
$$

for $\delta \leq 2^{-\pi \varepsilon^{-1}}$. Conversely, if $K$ is contained in an s-quasicircle with constant $\varepsilon$, then,

$$
\sup _{Q \in \mathcal{D}} \beta_{K}(Q) \leq C_{0} \varepsilon^{\frac{1}{2}}
$$

where $C_{0}$ is independent of $\varepsilon$.

REMARK. The best estimate for $\delta$ is not known. However, it is easy to see that Theorem 1.2 is not true if we replace $2^{-\pi \varepsilon^{-1}}$ by $\varepsilon^{n}$, for any $n \in \mathbb{Z}^{+}$.

The proof of Theorem 1.2 is a straight forward construction. We postpone it and present it in the last section, $\S 4$. According to Theorem 1.2, to prove Theorem I, we need only to consider the set $K$ which is a subset of an s-quasicircle with constant $\varepsilon$. Actually, we will see that Theorem $I$ is true for any set $K$ which is a subset of the quasicircle with constant $Q-1$ small, where $Q$ is the constant in (1.1) (see $\S 2)$. 
LEMMA 1.3. Suppose $\Gamma$ is a quasicircle with constant $Q$ and $K \subset \Gamma$ is compact. Then there is a Jordan curve $\Gamma_{0}$ such that $K \subset \Gamma_{0}$ and $\Gamma_{0} \backslash K=\cup \gamma_{j}, \gamma_{j}$ is chord-arc curve with the constant depending only on $Q$ and $\gamma_{j} \cap \Gamma=\left\{u_{j}, v_{j}\right\} \subset K$

Proof. Let $\Omega_{\Gamma}$ be one of components of $\mathbb{C} \backslash \Gamma$. Assume $\Phi(z)$ is a conformal mapping from the upper half-plane to $\Omega_{\Gamma}$. Note that $\Phi$ can be extended to a quasiconformal mapping, since $\Gamma$ is a quasicircle. Let $E=\Phi^{-1}(K)$. Since $K$ is closed set, $\mathbb{R} \backslash E=\cup I_{j}$, $I_{j}=\left(u_{j}, v_{j}\right)$. We choose $z_{j}=x_{j}+i y_{j}$, where $x_{j}=\left(u_{j}+v_{j}\right) / 2$ and $y_{j}=\left(v_{j}-u_{j}\right) / 2$. Let $\alpha_{j}=\left[u_{j}, z_{j}\right] \cup\left[z_{j}, v_{j}\right]$ and $\gamma_{j}=\Phi\left(\alpha_{j}\right)$. Finally, we set $\Gamma_{0}=\left\{\cup \gamma_{j}\right\} \cup K$.

It is clear that $\Gamma_{0}$ is a Jordan curve and it contains $K$. We can prove easily that $\gamma_{j}$ is a chord-arc curve with constant depending only on $Q$, by using the distortion theorem and the following result, due to Jerison and Kenig (see [17] for both results):

Proposition. If $\Gamma$ is a quasicircle with constant $Q$, then the image under $\Phi$ of a line $\left\{z=x+e^{i \theta} y: y>0\right\}, \theta \in(\pi / 8,7 \pi / 8)$, is a chord-arc curve with estimate constant depending only on $Q$.

We should point out that the original result is stated only for vertical lines, i.e., $\theta=\frac{\pi}{2}$, but the proof in $[\mathbf{1 7}]$ is valid for the lines in this Proposition.

We will use the following elementary geometric property of quasicircles:

Lemma 1.4. Suppose $\Gamma$ is a quasicircle with constant $Q, Q=$ $1+\varepsilon, \varepsilon<\frac{1}{10}$ and $z_{1}, z_{2} \in \Gamma$. Then there is $\theta \in(0,2 \pi)$ such that

$$
\Gamma\left(z_{1}, z_{2}\right) \backslash D\left(z_{1}, 10 \varepsilon r\right) \subset\left\{z: \Im\left(e^{i \theta}\left(z-z_{1}\right)\right)>\frac{\left|z-z_{1}\right|}{15}\right\},
$$

where $r=\left|z_{1}-z_{2}\right|$. and $\Gamma\left(z_{1}, z_{2}\right)$ is the smaller arc of $\Gamma$ between $z_{1}$ and $z_{2}$.

Without loss of generality we can assume $z_{1}=0, z_{2}=i r$. Let

$$
\begin{aligned}
& \mathcal{S}_{0}=\left\{z \in \mathbb{C}:-\frac{\pi}{20} \leq \arg (z) \leq \frac{5}{6} \pi\right\}, \\
& \mathcal{S}_{1}=\left\{z \in \mathbb{C}: \frac{\pi}{6} \leq \arg (z) \leq \frac{21}{20} \pi\right\} .
\end{aligned}
$$


Using the three points condition (1.1) we can easily prove $\Gamma\left(z_{1}, z_{2}\right) \backslash$ $D\left(z_{1}, 10 \varepsilon r\right) \subset \mathcal{S}_{0} \cup \mathcal{S}_{1}$. By a simple calculation, for $z \in \mathcal{S}_{0}$,

$$
\Im\left(e^{i \frac{\pi}{12}} z\right) \geq|z| \sin \left(\frac{\pi}{30}\right)>\frac{1}{15}|z|
$$

and, for $z \in \mathcal{S}_{1}$,

$$
\Im\left(e^{-i \frac{\pi}{12}} z\right) \geq|z| \sin \left(\frac{\pi}{30}\right)>\frac{1}{15}|z| .
$$

Thus, it is enough to show $\Gamma\left(z_{1}, z_{2}\right) \backslash D\left(z_{1}, 10 \varepsilon r\right)$ is a subset of either $\mathcal{S}_{0}$ or $\mathcal{S}_{1}$.

Claim. If there exists a $z \in \Gamma\left(z_{1}, z_{2}\right) \backslash D\left(z_{1}, 10 \varepsilon r\right)$ satisfying

$$
\frac{5 \pi}{6}<\arg (z)<\frac{21 \pi}{20}
$$

then

$$
\Gamma\left(z_{1}, z_{2}\right) \backslash D(0,10 \varepsilon r) \subset \mathcal{S}_{1}
$$

Actually, if there is no $z \in \Gamma\left(z_{1}, z_{2}\right) \backslash D\left(z_{1}, 10 \varepsilon r\right)$ satisfying (1.4), then

$$
\Gamma\left(z_{1}, z_{2}\right) \backslash D\left(z_{1}, 10 \varepsilon r\right) \subset S_{0}
$$

otherwise, by the claim,

$$
\Gamma\left(z_{1}, z_{2}\right) \backslash D\left(z_{1}, 10 \varepsilon r\right) \subset S_{1}
$$

Thus, Lemma 1.4 follows once our claim is proved.

Proof of Claim. Let $\zeta \in \Gamma\left(z_{1}, z_{2}\right)$ with $|\zeta| \geq 10 \varepsilon r$. Then $-\pi / 20 \leq$ $\arg (\zeta) \leq 21 \pi / 20$. Assume $\arg (\zeta)<\pi / 6$. Let $\theta=|\arg (\zeta)-\arg (z)|$, then $2 \pi / 3<\theta<11 \pi / 10$. Thus,

(1.5) $|z-\zeta|^{2}=|z|^{2}+|\zeta|^{2}-2|z||\zeta| \cos \theta \geq|z|^{2}+|\zeta|^{2}+|z||\zeta|$.

If $\zeta$ is between $z_{1}$ and $z$, using the three points condition, $|z-\zeta|<$ $(1+\varepsilon)|z|$. But by $(1.4),(1.5)$ and $\varepsilon<1$,

$$
|z-\zeta|>\left(|z|+\frac{|\zeta|}{2}\right)>|z|\left(1+\frac{5}{2} \varepsilon\right)
$$


which is a contradiction. If $\zeta$ is between $z$ and $z_{2}$, using the three points condition, $|z-\zeta|<(1+\varepsilon)|\zeta|$. Again by (1.4), (1.5) and $\varepsilon<1$,

$$
|z-\zeta|>\left(|\zeta|+\frac{|z|}{2}\right)>|\zeta|\left(1+\frac{5}{2} \varepsilon\right),
$$

which is impossible. Thus, $\arg (\zeta) \geq \frac{\pi}{6}$. Therefore, the claim is proved.

Let $\Gamma$ be a quasicircle with constant $1+\varepsilon$. We pick two points $a_{i}, b_{i} \in \Gamma$ and define $r_{i}=\left|a_{i}-b_{i}\right|$ and $c_{i}=\left(a_{i}+b_{i}\right) / 2$. We choose two other points $a_{j}, b_{j} \in \Gamma$ which are contained in the same component of $\Gamma \backslash \Gamma\left(a_{i}, b_{i}\right)$, and define $r_{j}=\left|a_{j}-b_{j}\right|$ and $c_{j}=\left(a_{j}+b_{j}\right) / 2$.

Lemma 1.5. Suppose $\varepsilon<\frac{1}{4}$. Then the following must be true that

$$
D\left(c_{i}, \frac{r_{i}}{100}\right) \bigcap D\left(c_{j}, \frac{r_{j}}{100}\right)=\phi, \quad i \neq j .
$$

Proof. By the three points condition (1.1),

$$
r_{i}=\left|a_{i}-b_{i}\right|<(1+\varepsilon) \min \left\{\left|a_{i}-a_{j}\right|,\left|a_{i}-b_{j}\right|\right\} .
$$

Without lose of generality we assume $r_{j} \leq r_{i}$ and $\left|a_{i}-a_{j}\right| \leq$ $\left|a_{i}-b_{j}\right|$ and $b_{i}$ separates $a_{i}$ from $a_{j}$. Then, by a simple geometric observation, and the Pythagorean theorem,

$$
\left|a_{i}-c_{j}\right|^{2} \geq\left|a_{i}-a_{j}\right|^{2}-\left|c_{j}-a_{j}\right|^{2} .
$$

Then, by (1.5) and $\varepsilon<\frac{1}{4}$,

$$
\begin{aligned}
\left|a_{i}-c_{j}\right| & \geq r_{i} \sqrt{\frac{1}{(1+\varepsilon)^{2}}-\frac{1}{4}} \\
& \geq \frac{3}{5} r_{i} .
\end{aligned}
$$

Using this estimate, we have

$$
\begin{aligned}
\left|c_{i}-c_{j}\right| & \geq\left|a_{i}-c_{j}\right|-\left|a_{i}-c_{i}\right| \\
& \geq \frac{1}{10} r_{i} .
\end{aligned}
$$

Therefore, $D\left(c_{i}, \frac{1}{100} r_{i}\right) \cap D\left(c_{j}, \frac{1}{100} r_{j}\right)=\phi$. 


\section{Proof of Theorem I.}

The definition of the Cauchy integral operator on AD regular sets. We observe that an $\mathrm{AD}$-regular set $\mathrm{K}$ with the Euclidean metric and Hausdorff measure is a space of homogeneous type [9]. We define the Cauchy integral operator as in [5]:

$$
\langle\mathcal{C} f, g\rangle=\frac{1}{2} \int_{K \times K} \frac{1}{\zeta-z}[f(\zeta) g(z)-f(z) g(\zeta)] d \Lambda(\zeta) d \Lambda(z),
$$

for $f, g$ Lipschitz functions. The integral converges absolutely because of the AD regularity of $K$ and the Lipschitz continuity of $f$ and $g$. Thus, $\mathcal{C}$ is well-defined as a continuous linear operator from Lipschitz functions into the class of distributions. It is easy to verify that the class of Lipschitz functions is dense in $L^{2}(K)$. We may formally write

$$
\mathcal{C} f(x)=\int_{K} \frac{f(\zeta)}{\zeta-z} d \Lambda(\zeta)
$$

We also need the following definitions

$$
\mathcal{C}^{\varepsilon} f(x)=\int_{\substack{|x-y|>\varepsilon \\ y \in K}} f(y)(x-y)^{-1} d \Lambda(y)
$$

and

$$
\mathcal{C}^{*} f(x)=\sup _{\varepsilon>0}\left|\mathcal{C}^{\varepsilon} f(x)\right|
$$

By a standard argument we have

Proposition 1.0. [5]. Let $K$ be $A D$ regular. Then the following are equivalent:

(i) $\mathcal{C}$ is bounded on $L^{2}(K)$,

(ii) $\mathcal{C}^{\varepsilon}$ is bounded on $L^{2}(K)$, uniformly in $\varepsilon$,

(iii) $\mathcal{C}^{*}$ is bounded on $L^{2}(K)$.

Proof of Theorem I. Suppose that $K \subset \mathbb{C}$ is AD-regular with constant $M$ (see the introduction) and

$$
\beta(K)<\delta .
$$

By Theorem 1.2, (2.1) implies $K \subset \Gamma$, where $\Gamma$ is an s-quasicircle with constant $\varepsilon$ (where $\varepsilon$ is sufficient small as long as $\delta$ is small 
enough). Thus, Theorem I will be included in the following slightly more general result:

THEOREM 2.1. Suppose $K$ is AD-regular with constant $M$ and the Cauchy integral operator is bounded on $L^{2}(K)$. Further suppose that $K$ is contained in a quasicircle with constant $1+\varepsilon$, where $\varepsilon>0$ is small enough, depending only on $M$ and $\|\mathcal{C}\|$. Then $K$ is contained in a chord-arc curve.

Proof. By Proposition 1.0, $\mathcal{C}^{*}$ is also bounded on $L^{2}(K)$. Using Lemma 1.3, we obtain a Jordan curve $\Gamma_{0}$ satisfying $\Gamma_{0} \backslash K=\cup \gamma_{j}, \gamma_{j}$ is a chord-arc curve with constant depending only on $\varepsilon$. To verify $\Gamma_{0}$ is chord-arc curve, we take any two points $x_{1}$ and $x_{2}$ on $\Gamma_{0}$ and we need to show

$$
\Lambda\left(\Gamma_{0}\left(x_{1}, x_{2}\right)\right) \leq C_{0}\left|x_{1}-x_{2}\right| .
$$

Let $\mathcal{G}=\left\{\gamma_{j}: \gamma_{j} \subset \Gamma_{0}\left(x_{1}, x_{2}\right)\right\}$. Then, by the three points condition (1.1) and AD-regularity, it is easy to see that, to show (2.2), it suffices to prove

$$
\sum_{\gamma_{j} \in \mathcal{G}} \Lambda\left(\gamma_{j}\right) \leq C_{0}\left|x_{1}-x_{2}\right|
$$

By Lemma $1.3, \gamma_{j} \cap K=\left\{a_{j}, b_{j}\right\}$, let $I_{j}=\left[a_{j}, b_{j}\right]$, then

$$
\Lambda\left(\gamma_{j}\right) \leq C_{0} \Lambda\left(I_{j}\right)
$$

Let $r=\left|x_{1}-x_{2}\right|$. Thus, in order to prove (2.3), we need to show

$$
\sum_{\gamma_{j} \in \mathcal{G}} \Lambda\left(I_{j}\right) \leq C_{0} r
$$

To establish (2.4), we introduce the following definition:

Definition 2.2. Suppose $\mathcal{P}=\{I\}$ is a collection of sets and $\rho>0$. A subcollection $\left\{I_{p, q}\right\}$ of $\mathcal{P}$ is called a $\rho$-frame of $\mathcal{P}$, if it satisfies the following conditions:

(i) There are pairwise disjoint subcollections $\mathcal{P}_{p, q}$ of $\mathcal{P}$ such that

$$
\bigcup_{p, q} \mathcal{P}_{p, q}=\mathcal{P}
$$


and, for each index pair $(p, q)$,

$$
\sum_{I \in \mathcal{P}_{p, q}} \Lambda(I) \leq C_{1} \Lambda\left(I_{p, q}\right)
$$

where $C_{1}$ is independent of $p, q$ and $\rho$.

(ii) There is a constant $C_{2}$ independent of $\rho$ such that

$$
\sum_{p, q} \Lambda\left(I_{p, q}\right) \leq C_{2} \rho
$$

It follows immediately from the definition of a $\rho$-frame that

$$
\sum_{I \in \mathcal{P}} \Lambda(I) \leq C_{1} C_{2} \rho
$$

Let $\mathcal{P}=\left\{I_{j}\right\}$. Hence, our problem is reduced to finding an $r$ frame of $\mathcal{P}$. Let $I_{j}=\left[a_{j}, b_{j}\right], \ell\left(I_{j}\right)=\left|b_{j}-a_{j}\right|$, and let $\Delta\left(I_{j}\right)=$ $D\left(a_{j}, \varepsilon_{j}\right), \varepsilon_{j}=\varepsilon \ell\left(I_{j}\right)$. Also, for simplicity, we may assume $\mathcal{P}$ is a finite set, since our estimate is independent of the number of elements in $\mathcal{P}$.

To find the $r$-frame of $\mathcal{P}$, we choose $I_{1,1} \in \mathcal{P}$ so that $\ell\left(I_{1,1}\right)$ is the maximum of the lengths of the $I_{j}$. Let

$$
\mathcal{P}_{1,1}=\left\{I_{j} \in \mathcal{P}: \ell\left(I_{j}\right) \geq \varepsilon \ell\left(I_{1,1}\right) \text { and } I_{j} \cap \Delta\left(I_{1,1}\right) \neq \phi\right\},
$$

and $\bar{\Delta}\left(I_{1,1}\right)=2 \Delta\left(I_{1,1}\right)$. Define

$$
\mathcal{I}_{1,1}=\left\{I_{j} \in \mathcal{P}: I_{j} \subset \bar{\Delta}\left(I_{1,1}\right)\right\} \backslash \mathcal{P}_{1,1}
$$

Assume we have chosen $I_{1, j}, \mathcal{P}_{1, j}$ and $\mathcal{I}_{1, j}, 1 \leq j \leq \nu$. Choose

$$
I_{1, \nu+1} \in \mathcal{P} \backslash \bigcup_{j=1}^{\nu}\left(\mathcal{P}_{1, j} \cup \mathcal{I}_{1, j}\right)
$$

which is of maximal possible length. Let

$$
\begin{aligned}
\mathcal{P}_{1, \nu+1}=\left\{I_{j} \in \mathcal{P} \backslash \bigcup_{j=1}^{\nu}\left(\mathcal{P}_{1, j} \cup \mathcal{I}_{1, j}\right): \ell\left(I_{j}\right)\right. & \\
& \left.\geq \varepsilon \ell\left(I_{1, \nu+1}\right), I_{j} \cap \Delta\left(I_{1, \nu+1}\right) \neq \phi\right\}
\end{aligned}
$$


and let

$$
\mathcal{I}_{1, \nu+1}=\left\{I_{j} \in \mathcal{P} \backslash \bigcup_{j}^{\nu}\left(\mathcal{P}_{1, j} \cup \mathcal{I}_{1, j}\right): I_{j} \subset \bar{\Delta}\left(I_{1, \nu+1}\right)\right\} \backslash \mathcal{P}_{1, \nu+1} .
$$

After finitely many steps, this procedure will stop. We obtain subcollections $\left\{\mathcal{P}_{1, j}\right\}$ which are disjoint from the elements of $\left\{I_{1, j}\right\}$.

Let $\mathcal{I}_{1}=\bigcup_{j} \mathcal{I}_{1, j}$. We repeat the same procedure as before to obtain $\mathcal{P}_{2, j}$ and $\mathcal{I}_{2, j}$. Let $\mathcal{I}_{2}=\bigcup_{j} \mathcal{I}_{2, j}$ and keep on going until $\mathcal{P}$ exhausted. Thus, $\left\{I_{p, q}\right\}$ and $\left\{\mathcal{P}_{p, q}\right\}$ are constructed.

We have to check that $\left\{I_{p, q}\right\}$ is a $r$-frame of $\left\{I_{j}\right\}$. By the construction of $\left\{\mathcal{P}_{p, q}\right\}$, it is clear that $\mathcal{P}=\cup_{p, q} \mathcal{P}_{p, q}$. We verify (2.5). For each index pair $(p, q)$, by the construction of $\mathcal{P}_{p, q}$, we have

$$
\varepsilon \ell\left(I_{p, q}\right) \leq \ell(I) \leq \ell\left(I_{p, q}\right), \quad \forall I \in \mathcal{P}_{p, q} .
$$

Let $I_{p, q}=\left[a_{p, q}, b_{p, q}\right]$. Since $K$ is in a subset of the quasicircle with constant $1+\varepsilon$, by Lemma $1.5, \forall I_{i}, I_{j} \in \mathcal{P}_{p, q}$,

$$
D\left(c_{i}, \frac{1}{100} \ell\left(I_{j}\right)\right) \cap D\left(c_{j}, \frac{1}{100} \ell\left(I_{j}\right)\right)=\phi, \quad i \neq j
$$

where $c_{j}$ is the center of $I_{j}$. So

$$
\sum_{I_{j} \in \mathcal{P}_{p, q}} \ell\left(I_{j}\right) \leq \frac{100^{2}}{\pi \varepsilon \ell\left(I_{p, q}\right)} \sum_{I_{j} \in \mathcal{P}_{p, q}} \pi\left(\frac{\ell\left(I_{j}\right)}{100}\right)^{2} \leq \frac{100^{2}}{\varepsilon} \ell\left(I_{p, q}\right) .
$$

Here, we used $I_{j} \cap \Delta\left(I_{p, q}\right) \neq \phi$, which implies $D\left(c_{j}, \frac{1}{100} \ell\left(I_{j}\right)\right) \subset$ $D\left(a_{p, q}, \ell\left(I_{p, q}\right)\right)$. Thus, (2.5) is verified, and, so, (i) is true.

Next, to verify (ii), we need to show (2.6). First, we note that for any fixed $p$,

$$
\frac{1}{2} \Delta\left(I_{p, q}\right) \cap \frac{1}{2} \Delta\left(I_{p, q^{\prime}}\right)=\phi
$$

if $q \neq q^{\prime}$ and, for any $I_{p, q}$, there exists $I_{p-1, q^{\prime}}$, such that

$$
I_{p, q} \subset \bar{\Delta}\left(I_{p-1, q^{\prime}}\right) \text {. }
$$

We claim that

$$
(2.9) K \cap \Delta\left(I_{p, q}\right) \subset\left\{x \in K: \mathcal{C}^{*}\left(\mathcal{X}_{\overline{\bar{\Delta}}\left(I_{p-1, q^{\prime}}\right)}\right)(x)>c_{0} \log \varepsilon^{-1}\right\}
$$


By the definition of $\mathcal{C}^{*}$, we can see that it is enough to prove our claim to show:

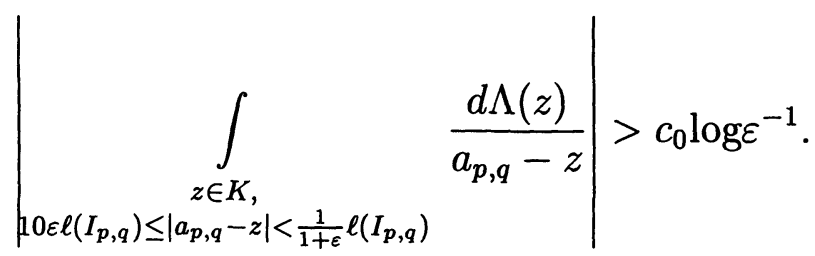

Let $\Gamma_{a_{p, q}}$ be the component of $\Gamma \backslash\left\{a_{p, q}\right\}$ which does not contain $b_{p, q}$. We choose $x \in \Gamma_{a_{p, q}}$ such that $\left|a_{p, q}-x\right|=\ell\left(I_{p, q}\right)$. By Lemma 1.4, there is $\theta \in[0,2 \pi)$ such that

$$
\begin{aligned}
\Gamma\left(a_{p, q}, x\right) \backslash D\left(a_{p, q}, 10 \varepsilon \ell\left(I_{p, q}\right)\right) & \subset S\left(a_{p, q}\right) \\
= & \left\{z: \Im\left\{e^{i \theta}\left(z-a_{p, q}\right)\right\}>\frac{\left|z-a_{p, q}\right|}{10}\right\}
\end{aligned}
$$

and using the three points condition (1.1), we have

$$
\begin{aligned}
\left\{z \in K: 10 \varepsilon \ell\left(I_{p, q}\right) \leq\left|a_{p, q}-z\right|\right. & \left.<\frac{\ell\left(I_{p, q}\right)}{1+\varepsilon}\right\} \\
& \subset \Gamma\left(a_{p, q}, x\right) \backslash D\left(a_{p, q}, 10 \varepsilon \ell\left(I_{p, q}\right)\right) .
\end{aligned}
$$

Therefore,

$$
\begin{aligned}
\mid & \int_{z \in K 10 \varepsilon \ell\left(I_{p, q}\right)} \leq\left|a_{p, q}-z\right|<\frac{1}{1+\varepsilon} \ell\left(I_{p, q}\right) \\
\geq & \frac{1}{10} \int_{\substack{z \in K \\
10 \varepsilon \ell\left(I_{p, q}\right) \leq\left|a_{p, q}-z\right|<\frac{1}{1+\varepsilon} \ell\left(I_{p, q}\right)}} \frac{d \Lambda(z)}{\left|a_{p, q}-z\right|} \\
& >c_{0} \log \varepsilon^{-1},
\end{aligned}
$$

where $c_{0}$ depends only on the AD-regularity constant $M$. Thus, we obtain (2.10) and, consequently, (2.9). Similarly, we can prove, for any $t$,

$$
K \cap \Delta\left(I_{1, t}\right) \subset\left\{x \in K: \mathcal{C}^{*}\left(\mathcal{X}_{D\left(a_{1,1}, 4 r\right) \cap K}\right)(x)>c_{0} \log \varepsilon^{-1}\right\}
$$


Because of $(2.7),(2.8),(2.9)$ and the $L^{2}$ boundedness of $\mathcal{C}^{*}$, we have the following estimates: for any fixed $p$,

$$
\begin{aligned}
\sum_{q: I_{p, q} \subset \bar{\Delta}\left(I_{p-1, q^{\prime}}\right)} & \Lambda\left(K \cap \frac{1}{2} \Delta\left(I_{p, q}\right)\right) \\
& \leq \Lambda\left\{x \in K: \mathcal{C}^{*}\left(\mathcal{X}_{\overline{\bar{\Delta}}\left(I_{p-1, q^{\prime}}\right)}\right)(x)>c_{\circ} \log \varepsilon^{-1}\right\} \\
& \leq \frac{1}{\left(c_{\circ} \log \varepsilon^{-1}\right)^{2}} \int_{K}\left(\mathcal{C}^{*}\left(\mathcal{X}_{\overline{\bar{\Delta}}\left(I_{p-1, q^{\prime}}\right)}\right)\right)^{2} d \Lambda(x) \\
& \leq \frac{\varepsilon \ell\left(I_{p-1, q^{\prime}}\right)}{4 M}\left\|\mathcal{C}^{*}\right\|^{2}
\end{aligned}
$$

if we choose $\varepsilon$ small enough, i.e., $\varepsilon$ small depending only on $\mathrm{M}$ and $\left\|\mathcal{C}^{*}\right\|$. Since $\mathrm{K}$ is $\mathrm{AD}$ regular, for any fixed $p$

$$
\sum_{q: I_{p, q} \subset \bar{\Delta}\left(I_{p-1, q^{\prime}}\right)} \ell\left(I_{p, q}\right) \leq \frac{1}{2} \ell\left(I_{p-1, q^{\prime}}\right) .
$$

Iterating the above inequality and using (2.11), we obtain the following:

$$
\begin{aligned}
\sum_{q} \ell\left(I_{p, q}\right) & \leq \frac{1}{2} \sum_{q^{\prime}} \ell\left(I_{p-1, q^{\prime}}\right) \\
& \leq \frac{1}{2^{p-1}} \sum_{t} \ell\left(I_{1, t}\right) \\
& \leq \frac{C\left(M,\left\|\mathcal{C}^{*}\right\|\right)}{2^{p}} r .
\end{aligned}
$$

Thus, (2.6) is verified by summing over $p$. This completes the proof of Theorem 2.1, and, thus, Theorem I is proved, assuming Theorem 1.2 which will be proved in Section 4 .

The author thanks Professor P. Jones for suggesting the use of weak type estimates in the proof of Theorem 1.2.

\section{The Cauchy Integral Operator And Analytic Capac- ity.}

Basic geometric theory. Let us recall some basic definitions and results from geometric measure theory that we will use. 
DEFINITION 3.1. The upper and lower densities of a closed onedimensional set $E$ at a point $x$ are defined by

$$
\begin{aligned}
& \bar{D}(E, x)=\limsup _{r \rightarrow 0} \frac{\Lambda(E \cap \overline{D(x, r)})}{2 r} \\
& \underline{D}(E, x)=\liminf _{r \rightarrow 0} \frac{\Lambda(E \cap \overline{D(x, r)})}{2 r} .
\end{aligned}
$$

Definition 3.2. A one-dimensional set $E$ is said to be regular in the sense of Besicovitch, or, simply, B-regular if

$$
\bar{D}(E, x)=\underline{D}(E, x)=1 \text {. }
$$

for a.e. $x \in E$. A point $x \in E$ satisfying the above equality is said to be regular; otherwise it is called B-irregular.

DEFINITION 3.3. A one-dimensional set is B-irregular if almost all of its points are B-irregular.

Given $x \in \mathbb{C}$, a line $L$ containing $x$ and $0<\theta<\frac{\pi}{4}$, we define the cone $S(x, L, \theta)$ to be the union of all lines passing through $x$ and making an angle of at most $\theta$ with $L$.

Definition 3.4. Let $E$ be a one-dimensional set and $x \in E$ a point at which $\bar{D}(E, x)>0$. Then a line $L$ passing through $x$ is tangent to $E$ at $x$ if

$$
\lim _{r \rightarrow 0} r^{-1} \Lambda([E \cap D(x, r)] \backslash S(x, L, \theta))=0
$$

for all $\theta>0$. $L$ is a weak tangent to $E$ at $\mathrm{x}$ if " $\lim _{r \rightarrow 0}$ " above is replaced by "im $r \rightarrow 0$ ".

Note that the tangent to $E$ expresses a certain smoothness of $E$, but the weak tangent does not contain any information of smoothness for $E$. We will present a class of sets $E$ at the end of this section that are B-irregular but there are weak tangents to $E$ at a.e. $x \in E$ in all directions.

The basic results in geometric measure theory we shall need are the following:

THEOREM 3.5. Let $E \subset \mathbb{C}$ be closed and have finite onedimensional Hausdorff measure. Then the following are equivalent: 
- $\quad E$ is regular in the sense of Besicovitch.

- $E$ has a tangent at a.e. $x \in E$.

- $\quad E$ is contained in a countable union of rectifiable curves.

ThEOREM 3.6. Let $E \subset \mathbb{C}$ be closed and have finite one-dimensional Hausdorff measure. Then the following are equivalent:

- $\quad E$ is irregular in the sense of Besicovitch.

- $\quad$ At a.e. $x \in E, E$ has no tangent.

- For any rectifiable curve $\Gamma, \Lambda(E \cap \Gamma)=0$.

For proof of these results see [13].

It is very important to characterize a set of positive analytic capacity in terms of the geometry of the set. The following theorem was established by Mattila [25]

THEOREM 3.7. Suppose $K$ is compact and B-irregular with $\Lambda(K)<\infty$ and suppose that for a.e. $x \in K$, there is at least one $L$ passing through $x$ which is not a weak tangent to $K$ at $x$. Then $\gamma(K)=0$.

Definition 3.8. Suppose $K \subset \mathbb{C}$ is compact and B-irregular. We say $K$ satisfies Mattila's condition if it satisfies the condition that for almost all $x \in K, \underline{D}(K, x)>0$ and there is no weak tangent to $E$ at $x$, at least in one direction.

Using this definition we can restate Theorem 3.7 equivalently as follows:

Theorem 3.7'. Suppose $\Lambda(K)<\infty$ and $K$ satisfies Mattila's condition. Then $\gamma(K)>0$ if and only if there is a rectifiable curve $\Gamma$ such that $\Lambda(\Gamma \cap K)>0$.

A geometric observation for locally flat sets. We note that a locally flat set is certainly not necessarily B-regular. The following result describes a geometrical property of locally flat sets:

Proposition 3.9. Suppose $K$ is AD-regular and locally flat. Then, for almost all points $x$, either there is a tangent to $K$ at $x$, or there are weak tangents to $K$ in all directions at $x$. In particular, if $K$ is not B-regular, $K$ does not satisfy Mattila's condition.

To prove Proposition 3.9, we will use the following two theorems of Besicovitch [13]: 
THEOREM 3.10. Suppose $K$ has finite one-dimensional Hausdorff measure. Then $K$ can be decomposed into two subsets $G$ and $B$ such that $G$ is $B$-regular and $B$ is B-irregular.

ThEOREM 3.11. Suppose $K$ is B-irregular. Then, given $\theta \in$ $[0,2 \pi), \phi \in(0, \pi / 2)$ and a line $L$ which contains $x$ and points in the direction $e^{i \theta}$,

(3.1) $\limsup _{r \rightarrow 0} r^{-1} \Lambda\left(K \cap D(x, r) \cap S^{+}(x, L, \phi)\right)$

$+\underset{r \rightarrow 0}{\limsup } r^{-1} \Lambda\left(K \cap D(x, r) \cap S^{-}(x, L, \phi)\right) \geq \frac{1}{6} \sin \phi, \forall$ a.e. $x \in K$,

where $S^{+}(x, L, \phi)$ is a one-way cone of $S(x, L, \phi)$ and $S^{-}(x, L, \phi)$ is the opposite one-way cone.

Proof of Proposition 3.9. By Theorem 3.10, $K=G \cup B$ where $G$ is B-regular and $B$ is B-irregular. By Theorem 3.1, there is a tangent to $K$ at a.e. $x \in G$. So we need only to show that there are weak tangents to $K$ in all directions at a.e. $x \in B$.

Without loss of generality we may assume $K$ is B-irregular. Let

$$
\theta_{p} \in[0,2 \pi) \quad \text { and } \quad \phi_{q} \in(0, \pi / 2)
$$

be all possible rational numbers. Set

$$
K_{p, q}=\left\{x \in K: \text { it satisfies (3.1) with } \theta_{p} \text { and } \phi_{q}\right\}
$$

and set

$$
K_{0}=\bigcap \lim _{p, q} K_{p, q}
$$

Since $\Lambda\left(K \backslash K_{p, q}\right)=0$,

$$
\Lambda\left(K \backslash K_{0}\right) \leq \sum_{p, q} \Lambda\left(K \backslash K_{p, q}\right)=0 .
$$

It suffices to show the following:

Claim. Any straight line $L$ which contains $x \in K_{0}$ is a weak tangent to $K$ at $x$.

Pick $\phi \in\left(0, \frac{\pi}{2}\right)$. Let $L$ be a line which points in the direction $\theta \in[0,2 \pi)$. Choose $(p, q)$ so that

$$
\left|\theta-\theta_{p}\right|<10^{-1000} \phi, \quad \text { and } \quad \phi_{p}<10^{-1000} \phi .
$$


Because $x \in K_{0}$, by Theorem 3.11 and (3.2), there are points

$$
x_{n} \in S\left(x, L, \frac{\phi}{500}\right) \cap K_{0}, \quad n=1, \cdots, \infty
$$

such that

$$
x_{n} \neq x_{m}, n \neq m \quad \text { and } \quad \lim _{n \rightarrow \infty} x_{n}=x .
$$

Let $r_{n}=\left|x-x_{n}\right|, \quad n=1,2, \cdots$. We choose $Q_{n} \in \mathcal{D}$ such that $3 Q_{n}$ contains both $x_{n}$ and $x$, and $Q_{n}$ has minimum possible size, i.e., $\ell\left(Q_{n}\right) \approx r_{n}$. Let $\beta_{K}\left(Q_{n}\right)=\delta_{n}$. Since $K$ is locally flat,

$$
\lim _{n \rightarrow \infty} \delta_{n}=0 \text {. }
$$

We may assume $\delta_{n} \leq \frac{\phi}{100}$. By (3.2)-(3.4) and an elementary calculation,

$$
\Lambda\left(K \cap D\left(x, a_{0} r_{n}\right) \backslash S(x, L, \phi)\right) \leq C(\phi) \delta_{n} r_{n},
$$

where $a_{0}$ is small independent constant, which implies

$$
\liminf _{r \rightarrow 0} r^{-1} \Lambda(K \cap D(x, r) \backslash S(x, L, \phi)) \leq C(\phi) \lim _{n \rightarrow \infty} \delta_{n}=0 .
$$

Thus, we showed that $L$ is a weak tangent to $K$ at $x$ and, therefore, Proposition 3.9 is proved.

Geometric characterization of analytic capacity of locally flat sets. By Proposition 2.9, locally flat sets do not satisfy Mattila's condition. So, Mattila's theorem doesn't work for locally flat sets. We now use Theorem I to study the analytic capacity of $K$. But, in general, knowing that $\gamma(K)>0$ tells us nothing about the $L^{2}$ boundedness of the Cauchy integral operators, because it could be that $K=A \cup B$ where $\gamma(A)>0$ and $\|\mathcal{C}\|$ gives no control on $B$. However, M. Christ [6] provided a useful tool:

THEOREM 3.12. Let $K \subset \mathbb{C}$ be AD-regular and suppose that $\gamma(K)>0$. Then there exists an AD-regular set $K^{\prime}$ such that $\mathcal{C}$ is bounded on $L^{2}\left(K^{\prime}\right)$ and $\Lambda\left(K \cap K^{\prime}\right)>0$.

REMARK. In particulary, if $K$ is locally flat, we will show the $K^{\prime}$ can be constructed to be locally flat. 
In particular, Theorem I together with Theorem 3.12 give us basic tools to study analytic capacity of locally flat sets. Because Theorem 3.12 is very important to us and its original proof is contained in a proof of a more general result (see [6] for details), we shall give, for reader's convenience, the details of a direct proof which contains a proof of the above Remark. The main idea of this proof is due to M. Christ. We begin by recalling some definitions and theorems (all of them can be found in [6]).

Definition 3.13. Suppose $K$ is AD-regular. Then a family of set $Q_{j}^{k} \subset K$ is called a dyadic system if there exist constants $r, \alpha>0, a_{0}, A_{0}<\infty$ such that

(i) $\Lambda\left(K \backslash \cup_{j} Q_{j}^{k}\right)=0, \quad$ for each $k$.

(ii) For any, $i, j, l, k$ with $l \geq k$, either $Q_{i}^{l} \subset Q_{j}^{k}$ or, $Q_{i}^{l} \cap Q_{j}^{k}=$ $\phi$.

(iii) For each $(k, j)$ and each $l, l<k$ there is a unique $i$ such that $Q_{j}^{k} \subset Q_{i}^{l}$.

(iv) Diameter $\left(Q_{j}^{k}\right) \leq A_{0} r^{k}$.

(v) Each $Q_{j}^{k}$ contains some disk $D\left(x_{j}^{k}, a_{0} r^{k}\right)$.

(vi) $\Lambda\left\{y \in Q_{j}^{k}: \operatorname{dist}\left(y, K \backslash Q_{j}^{k}\right) \leq t r^{k}\right\} \leq A_{0} t^{\alpha} \Lambda\left(Q_{j}^{k}\right), \quad \forall j, \quad k$. See [6] for the existence of a dyadic system for an AD-regular set. Also, following the terminology used in [6], we call $Q_{j}^{k}$ a dyadic cube. (Note that $Q_{j}^{k}$ is a subset of $K$ and is not a dyadic square.)

REMARK. The proof of existence of a dyadic system in [6] shows that any dyadic cube is itself an AD-regular set.

Let $\mathcal{D}(K)$ be a dyadic system for $K$.

Definition 3.14. A function $b \in L^{\infty}(K)$ is said to be dyadic para-accretive if for every $Q_{j}^{k} \in \mathcal{D}(K)$, there exists $Q_{i}^{l} \in \mathcal{D}(K), Q_{i}^{l} \subset$ $Q_{j}^{k}$, with $l \leq k+N$ and

$$
\left|\int_{Q_{i}^{l}} b d \Lambda\right| \geq c \Lambda\left(Q_{i}^{l}\right),
$$

for some fixed $c>0, N<\infty$.

DEFINITION 3.15. A locally integrable function $f$ belongs to dyadic $B M O$ if

$$
\sup _{Q} \inf _{C_{Q}} \Lambda(Q)^{-1} \int_{Q}\left|f(z)-C_{Q}\right| d \Lambda(z)<\infty
$$


where the supremum is taken over all dyadic cubes.

We will use the following $T(b)$ theorem:

THEOREM 3.16. Suppose that $b$ is a dyadic para-accretive function on $K$ and that $\mathcal{C}(b)$ is in dyadic $B M O$. Then $\mathcal{C}$ is bounded on $L^{2}(K)$.

Theorem 3.16 is a special version of a general $T(b)$ theorem, which suffices for our purpose (see [6] Theorem 20, for this general $T(b)$ theorem).

Proof of Theorem 3.12. It is well-know that if $\gamma(K)>0$ then there is $f \in H^{\infty}(\overline{\mathbb{C}} \backslash K)$ satisfying $\|f\|_{\infty} \leq 1, f(\infty)=0$ and $f^{\prime}(\infty) \neq 0$. In fact, we can write $f$ as the Cauchy integral of an $L^{\infty}$ function $g$ supported on $K$ satisfying $\|g\|_{\infty} \leq 1$ and $\int_{K} g d \Lambda \neq 0$.

Let $\gamma(K)=c_{0} \Lambda(K), c_{0}>0$. Assume $K \in \mathcal{D}(K)$, denote it by $Q_{1}^{1}$, and run a stopping-time procedure on it as follows: for $Q_{j}^{2} \in \mathcal{D}(K)$, which is $Q_{1}^{1}$ 's child, we stop the construction, if it satisfies

$$
\left|\int_{Q_{j}^{2}} g d \Lambda\right|<\frac{1}{2} c_{0} \Lambda\left(Q_{j}^{2}\right),
$$

i.e. $Q_{j}^{2}$ is a stopping time cube and we put this $Q_{j}^{2}$ aside. If it doesn't satisfy the above inequality, i.e.,

$$
\left|\int_{Q_{j}^{2}} g d \Lambda\right| \geq \frac{1}{2} c_{0} \Lambda\left(Q_{j}^{2}\right),
$$

we turn to the next generation, and repeat this argument. We keep this argument going on until it goes through all levels. Let $\mathcal{S}$ be the collection of all stopping time cubes. It is clear that the stopping time cubes are disjoint and satisfy (3.5). Also, it is easy to show

$$
\Lambda\left(K \backslash\left(\cup_{S \in \mathcal{S}} S\right)\right)>\frac{1}{2} c_{0} \Lambda(K)>0 .
$$

To construct $K^{\prime}$, we need to replace $S$ by a certain good set, $\Gamma_{S}$. By (iv) and (v) under Definition 3.13, for any $S \in \mathcal{S}, S$ contains a disk $D\left(x_{S}, d_{S}\right)$ satisfying $x_{S} \in S$ and $d_{S}>c_{1} \operatorname{diam}(S)$, where $c_{1}$ depends only on $a_{0}$ and $A_{0}$, and, thus, we can choose $z_{1}, z_{2} \in$ $D\left(x_{S}, d_{S}\right) \cap S$ such that

(3.8) $\left|z_{1}-z_{2}\right| \geq c_{2} d_{S} \quad$ and $\quad \operatorname{dist}\left(\left[z_{1}, z_{2}\right], K \backslash S\right) \geq c_{2} d_{S}$, 
where $c_{2}$ depends only on $M$ and $c_{1}$.

Let $c_{S}=\left(z_{1}+z_{2}\right) / 2, I_{S}=\left[z_{1}, c_{S}\right]$ and $J_{S}=\left[c_{S}, z_{2}\right]$. Let $\Gamma_{S}=$ $I_{S} \cup J_{S}$. Define

$$
K^{\prime}=\left(K \backslash \cup_{S \in \mathcal{S}} S\right) \bigcup\left(\cup_{S \in \mathcal{S}} \Gamma_{S}\right)
$$

By (3.7), $\Lambda\left(K \cap K^{\prime}\right)>0$ and, using the Remark below Definition 3.13 and (3.8), we can easily show that $K^{\prime}$ is AD-regular.

We now prove $K^{\prime}$ is locally flat. It suffices to show that, for any $Q \in \mathcal{D}$, there is $D Q \in \mathcal{D}$ such that $Q \subset D Q, \quad \ell(D Q)<C_{2} \ell(Q)$ and

$$
\beta_{K^{\prime}}(Q) \leq C_{3} \beta_{K}(D Q)
$$

where $C_{2}$ and $C_{3}$ depend only on $M, a_{0}$ and $A_{0}$. Let

$$
P_{Q}=\left\{\Gamma_{S}: 3 Q \cap \Gamma_{S} \neq \phi \quad \text { and } \quad 3 Q \cap\left(K^{\prime} \backslash \Gamma_{S}\right) \neq \phi\right\}
$$

Let

$$
E_{Q}=\cup\left\{\Gamma_{S}: \Gamma_{S} \in P_{Q}\right\}
$$

It is clear that, if $P_{Q}=\phi$, then either $3 Q \cap K^{\prime} \subset \Gamma_{S}$ or, $3 Q \cap K^{\prime} \subset$ $K \backslash \cup_{S \in \mathcal{S}} S$. But, in both cases, we have $\beta_{K^{\prime}}(Q) \leq \beta_{K}(Q)$, and we choose $D Q=Q$. We now assume $P_{Q} \neq \phi$. Note, for any $\Gamma_{S} \in P_{Q}$, we can find $a \in 3 Q \cap \Gamma_{S}$, and $b \in 3 Q \cap\left(K^{\prime} \backslash \Gamma_{S}\right)$. By the choice of $\Gamma_{S}, S \in \mathcal{S}$, it is clear that, there is a constant $C$ depending only on $M, c_{0}$ and $A_{0}$ such that

$$
\Lambda\left(\Gamma_{S}\right) \leq C \ell(Q)
$$

which yields $\Gamma_{S} \subset 10 C Q$. We choose a dyadic square $D Q$ such that

$$
10 C Q \subset 3 D Q, \quad \text { and } \quad \ell(D Q) \leq 10 \ell(10 C Q) .
$$

Then, we have

$$
\sup _{x \in\left(3 Q \cup K^{\prime}\right) \cup E_{Q}} \operatorname{dist}(x, L) \leq \sup _{y \in 3 D Q \cap K} \operatorname{dist}(y, L),
$$

for any straight line $L$, and, thus, by the definition of $\beta_{K}(Q)$, this yields,

$$
\beta_{K^{\prime}}(Q) \leq \frac{\ell(D Q)}{\ell(Q)} \beta_{K}(D Q) \leq 100 C \beta_{K}(D Q)
$$


Hence $K^{\prime}$ is locally flat.

By Theorem 3.16, to prove that the Cauchy integral operator is $L^{2}$ bounded on $K^{\prime}$, we need only to find a dyadic para-accretive function $b$ such that $\mathcal{C}(b)$ is a dyadic BMO function defined on $K^{\prime}$. To construct $b$, we recall that, for any $S \in \mathcal{S}, \Gamma_{S}=I_{S} \cup J_{S}$, $I_{S}=\left[z_{1}, c_{S}\right]$ and $J_{S}=\left[c_{S}, z_{2}\right]$, where $z_{1}, z_{2} \in S$ and $c_{S}=\left(z_{1}+z_{2}\right) / 2$. By (3.8), it is clear that

$$
\Lambda(S) \leq C_{4} \Lambda\left(J_{S}\right)
$$

where $C_{4}$ is independent of $S$. We define a piecewise constant function $g_{S}$ on $\Gamma_{S}$ by $g_{S}=C$ on $I_{S}$, and $g_{S}=-C+\frac{1}{\Lambda\left(J_{S}\right)} \int_{S} g d \Lambda$ on $J_{S}$, where $C=2 C_{4}$. By this definition, it is clear that

$$
\int_{\Gamma_{S}} g_{S} d \Lambda=\int_{S} g d \Lambda
$$

and, on $\Gamma_{S}$,

$$
1<\left|g_{S}(x)\right|<C
$$

Let

$$
b= \begin{cases}g & \text { on } K^{\prime} \cap K \\ g_{s} & \text { on } \Gamma_{S}, \quad S \in \mathcal{S} .\end{cases}
$$

We show that $b$ is a dyadic para-accretive function.

First of all, we have to define a dyadic system $\mathcal{D}\left(K^{\prime}\right)$ associated to $K^{\prime}$. Let

$$
\mathcal{D}_{1}\left(K^{\prime}\right)=\left\{Q^{\prime}: Q^{\prime}=(Q \backslash \cup S) \cup\left(\cup_{S \subset Q} \Gamma_{S}\right), Q \in \mathcal{D}(K) \backslash \mathcal{S}\right\},
$$

which is called the dyadic cube class of type 1 . Since $\Gamma_{S}$ is an interval, we define by $\mathcal{D}\left(\Gamma_{S}\right)$ to be all bisecting subintervals of $\Gamma_{S}$. It is clear that $I_{S}$ and $J_{S}$ are in $\mathcal{D}\left(\Gamma_{S}\right)$. Let

$$
\mathcal{D}_{2}\left(K^{\prime}\right)=\cup_{S \in \mathcal{S}} \mathcal{D}\left(\Gamma_{S}\right),
$$

which is called the dyadic cube class of type 2. Define $\mathcal{D}\left(K^{\prime}\right)=$ $\mathcal{D}_{1}\left(K^{\prime}\right) \cup \mathcal{D}_{2}\left(K^{\prime}\right)$.

We choose $Q^{\prime} \in \mathcal{D}\left(K^{\prime}\right)$ which is of type 1 . Choose $Q \in \mathcal{K}$ associated to $Q^{\prime}$. Then $Q$ is not a stopping time cube. By (3.9) and (3.6),

$$
\left|\int_{Q^{\prime}} b d \Lambda\right|=\left|\int_{Q} g d \Lambda\right| \geq c_{0}^{\prime} \Lambda\left(Q^{\prime}\right)
$$


where $c_{0}^{\prime}$ is independent of $Q^{\prime}$. Choose $Q^{\prime} \in \mathcal{D}\left(K^{\prime}\right)$ which is of type 2, i.e. $Q^{\prime} \in \mathcal{D}\left(\Gamma_{S}\right)$ for some $S \in \mathcal{S}$. Let $R=Q^{\prime}$, if $Q^{\prime} \neq \Gamma_{S}$, and let $R=I_{S}$, if $Q^{\prime}=\Gamma_{S}$. Since either $R \subset I_{S}$ or, $R \subset J_{S}$, by (3.10),

$$
\left|\int_{R} b d \Lambda\right|=\left|\int_{R} g_{S} d \Lambda\right| \geq \Lambda(R)
$$

Hence, $b$ is a dyadic para-accretive function.

To show that $b$ is a dyadic BMO function, it suffices to show that

$$
\int_{Q}\left|\mathcal{C}\left(b \chi_{Q}\right)\right| d \Lambda \leq C_{0} \Lambda(Q)
$$

Choose $Q^{\prime} \in \mathcal{D}\left(K^{\prime}\right)$. Note that, if $Q^{\prime}$ is of type 2 , say $Q^{\prime} \in \mathcal{D}\left(\Gamma_{S}\right)$, for some $S \in \mathcal{S}$, since the Cauchy integral operator is bounded on $L^{2}\left(I_{S}\right)$ with a constant independent of $S$, we can easily show (3.11) is true for $Q^{\prime}$. Thus, we need only to consider the case when $Q^{\prime}$ is of type 1 . The following two estimates will be used:

$$
\int_{Q}\left|\mathcal{C}\left(g \chi_{Q}\right)\right| d \Lambda \leq C_{5} \Lambda(Q)
$$

and

$$
\int_{Q \backslash S} \int_{S^{\prime}}\left|\frac{1}{x-y}-\frac{1}{x_{S}-y}\right| d \Lambda(x) d \Lambda(y) \leq C_{5} \Lambda(S),
$$

where $S^{\prime}$ is $S$ or $\Gamma_{S}$ and $x_{S}$ satisfies $\operatorname{dist}\left(x_{S}, Q \backslash S\right)>c \Lambda(S)$. They can be proved by using the fact that $|f(z)| \leq 1, \forall z \in \mathbb{C} \backslash K$ and (i)-(vi) in Definition 3.13. Since $Q^{\prime}$ is of type 1 , and by (3.9),

$$
\begin{aligned}
\mathcal{C}\left(b \chi_{Q}\right)(y)= & \int_{Q \backslash(\cup \mathcal{S})} \frac{g(x)}{x-y} d \Lambda(x)+\int_{\cup_{S \subset Q} \Gamma_{S}} \frac{g_{S}(x)}{x-y} d \Lambda(x) \\
= & \mathcal{C}\left(g \chi_{Q}\right)(y)+\sum_{S \subset Q} \int_{\Gamma_{S}} g_{S}(x)\left\{\frac{1}{x-y}-\frac{1}{x_{S}-y}\right\} d \Lambda(x) \\
& +\sum_{S \subset Q} \int_{S} g(x)\left\{\frac{1}{x_{S}-y}-\frac{1}{x-y}\right\} d \Lambda(x) .
\end{aligned}
$$


Hence, using (3.12) and (3.13), we have

$$
\begin{aligned}
& \int_{Q}\left|\mathcal{C}\left(b \chi_{Q}\right)(y)\right| d \Lambda(y) \\
& \leq C_{6} \Lambda(Q)+C_{6} \sum_{S \subset Q} \Lambda(S) \\
& \quad+\sum_{S \subset Q} \int_{Q \backslash S} \int_{\Gamma_{S}}\left|\frac{1}{x_{S}-y}-\frac{1}{x-y}\right| d \Lambda(x) d \Lambda(y) \\
& \quad+\sum_{S \subset Q} \int_{Q \backslash S} \int_{S}\left|\frac{1}{x_{S}-y}-\frac{1}{x-y}\right| d \Lambda(x) d \Lambda(y) \\
& \leq C_{7} \Lambda(Q)+C_{8} \sum_{S \subset Q} \Lambda(S) \\
& \leq C_{0} \Lambda(Q)
\end{aligned}
$$

and (3.11) is proved. Therefore, Theorem 3.12 is proved.

Proof of Theorem II. Since $K$ is AD-regular, without lose of generality, we may assume $K$ is of finite one-dimensional Hausdorff measure. By Theorem 3.12, we can find a locally flat $K^{\prime}$ such that $\Lambda\left(K^{\prime} \cap K\right)>0$ and $\mathcal{C}$ is bounded on $L^{2}\left(K^{\prime}\right)$. Fix an arbitrary small positive number $\delta$. We claim that $K=Z \cup\left(\cup_{j} K_{j}\right), \Lambda(Z)=0$ and, for each $j, K_{j}$ is AD-regular and satisfies

$$
\beta_{K_{j}}(Q) \leq \delta, \quad \forall Q \in \mathcal{D}
$$

Let $\mathcal{D}\left(K^{\prime}\right)=\left\{Q_{j}^{k}\right\}$. Let $Z_{k}=K^{\prime} \backslash\left(\cup_{j} Q_{j}^{k}\right)$ and $Z=\cup_{k} Z_{k}$. Since, by (i) of Definition 3.13, $\Lambda\left(Z_{k}\right)=0, \forall k, \Lambda(Z)=0$. For $x \in K^{\prime} \backslash Z$, there exits $\left\{Q_{j_{k}}^{k}\right\}$ such that $x \in \cap_{j} Q_{j_{k}}^{k}$, and, since $K^{\prime}$ is locally flat, there exits $Q_{j k_{x}}^{k_{x}}$ containing $x$ such that, $\forall Q \in \mathcal{D}$ satisfying

$$
\ell(Q)<\operatorname{diam}\left(Q_{j k_{x}}^{k_{x}}\right) \quad \text { and } \quad Q \cap Q_{j k_{x}}^{k_{x}} \neq \phi
$$

we have

$$
\beta_{K^{\prime}}(Q) \leq \delta .
$$

Letting $K_{x}=Q_{j_{k_{x}}}^{k_{x}}$, this yields

$$
\beta_{K_{x}}(Q) \leq \delta, \quad \forall Q \in \mathcal{D} .
$$

Since $K_{x}$ is a dyadic cube, $K_{x}$ is AD-regular by the Remark following Definition 3.13. Using a standard covering argument, we obtain 
our claim. Sine $\mathcal{C}$ is bounded on $L^{2}\left(K^{\prime}\right), \mathcal{C}$ is uniformly bounded on $L^{2}\left(K_{j}\right), \forall j$ with bound independent of $\delta$. Hence, if we choose $\delta$ to be small enough, then, by Theorem I, $K_{j}$ is a subset of the chord-arc curve $\Gamma_{j}$ for all $j$ (therefore, a subset of rectifiable curve). Hence, there must be $\Gamma_{j_{0}}$ such that $\Lambda\left(K \cap \Gamma_{j_{0}}\right)>0$. Theorem II is proved.

Corollary. If $K$ is locally flat, $\Lambda(K)<\infty$, and B-irregular, then $\gamma(K)=0$.

The proof of this corollary is obvious and we omit it.

An Example of Locally Flat Set: the Snowflake. The snowflake set $E$ constructed by G. David and S. Semmes (see [11], $135-137)$ is defined as follows: given a sequence $\left\{\alpha_{n}\right\}$ of small real numbers, say less than $\frac{1}{100}$, we construct $E_{n}$ recursively according to the following recipe. We take $E_{0}$ to be the unit interval on the x-axis. Suppose $E_{n-1}$ has been constructed. To construct $E_{n}$ we replace each line segment $L$ of $E_{n-1}$ by four segments $L_{1}, L_{2}, L_{3}$, $L_{4}$ with the following properties. (See Figure 0.)

(3.14) The length of $L_{i}$ is $4^{-n}, \quad i=1,2,3,4$.

(3.15) The endpoint of $L_{i}$ is the initial point of $L_{i+1}, \quad i=1,2,3$.

(3.16) The $L_{i}$ 's make the angles $0, \alpha_{n}, \pi-\alpha_{n}$, and 0 , respectively, with $L$.

(3.17) The midpoint of $L$ is also the midpoint of the segment that joins the initial point of $L_{1}$ to the endpoint of $L_{4}$.

Let $E$ be the limiting set of the $E_{n}$ 's in the Hausdorff metric. In [11] they prove the following properties of $E$ :

(i) $E$ is AD-regular.

(ii) $\quad E$ is B-regular iff $\sum \alpha_{n}^{2}<\infty$.

Note, using their construction of the snowflake set $E$, it not hard to see, if $\lim _{n \rightarrow \infty} \alpha_{n}=0, E$ is locally flat. So, we cannot use Mattila's theorem to characterize its analytic capacity. But, using Theorem II and (ii), we obtain, immediately, the following result: 


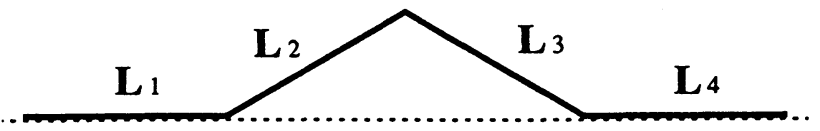

$\mathbf{L}$

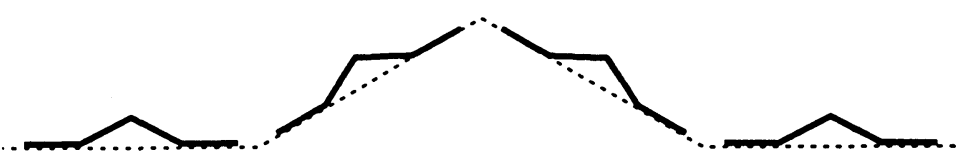

Figure $0 . \cdots$ the old curves

- the new curves.

Proposition 3.17. Suppose the snowflake set $E$ satisfys $\lim _{n \rightarrow \infty} \alpha_{n}=0$. Then $\gamma(E)>0$ iff $\sum \alpha_{n}^{2}<\infty$.

\section{A Characterization of Subsets of Some Qusiconformal} Circles. In this section we prove Theorem 1.2. Let $K$ be a compact set (that may not be AD-regular) in the complex plane which satisfies the condition in Theorem 1.1

$$
\beta(K) \leq \delta
$$

with $\delta=2^{-\pi \varepsilon^{-1}}$ and $\varepsilon<\frac{1}{100}$. Without loss of generality we can assume $K \subset[1 / 4,3 / 4] \times[1 / 4,3 / 4]$, because (4.0) and the three points condition are dilation and translation invariant.

Some lemmas. To construct an s-quasicircle with constant $\varepsilon$ containing $K$, we begin with some simple lemmas.

Lemma 4.1. Suppose $\beta(K) \leq \delta, \delta<\frac{1}{10}$ and suppose $x, y, z \in K$ satisfy

$$
|x-z| \geq \frac{1}{2} \max \{|x-y|,|y-z|\} .
$$

Then

$$
\operatorname{dist}(y, L)<148 \delta|x-z|
$$


where $L$ is the straight line containing $[x, z]$.

Proof. Let $|x-z| \in\left[2^{-k-1}, 2^{-k}\right]$. Choose $Q \in \mathcal{D}$ such that $\ell(Q)=$ $2^{-k+1}$ and $y \in Q$. It is clear that $\ell(Q) \leq 4|x-z|$. Since (4.1), $x, y, z \in 3 Q$. Let $L_{0}$ be the straight line such that

$$
\sup _{u \in 3 Q} \operatorname{dist}\left(u, L_{0}\right)=\beta_{K}(Q) \ell(Q)
$$

in particular,

$$
\operatorname{dist}\left(w, L_{0}\right) \leq \beta_{K}(Q) \ell(Q), \quad w=x, y, z .
$$

Let $L$ be the straight line containing $[x, z]$. If $L_{0}$ is parallel to $L$ it is easy to prove (4.2). Otherwise, let $L_{0} \cap L=\left\{x_{0}\right\}$. If $x_{0} \in$ $[x, z]$, without loss of generality we can assume $\left|x-x_{0}\right|<\mid x_{0}-$ $z \mid$ which implies $\left|x_{0}-z\right| \geq 1 / 2|x-z|$. If $x_{0} \notin[x, z], \max \{\mid x-$ $\left.x_{0}|,| x_{0}-z \mid\right\} \geq|x-z|$. Without loss of generality we can assume $\left|x_{0}-z\right| \geq 1 / 2|x-z|$. Thus, for $x_{l} \in 3 Q \cap L_{0}$,

$$
\begin{aligned}
\operatorname{dist}\left(x_{l}, L\right) & =\frac{\operatorname{dist}\left(z, L_{0}\right)}{\left|x_{0}-z\right|}\left|x_{0}-x_{l}\right| \\
& \leq \frac{\beta_{K}(Q) \ell(Q)}{\frac{1}{8} \ell(Q)} 3 \sqrt{2} \ell(Q) \\
& \leq 144 \delta|x-z| .
\end{aligned}
$$

Therefore,

$$
\begin{aligned}
\operatorname{dist}(y, L) & \leq \operatorname{dist}\left(y, L_{0}\right)+\sup _{x_{l} \in 3 Q \cap L_{0}} \operatorname{dist}\left(x_{l}, L\right) \\
& \leq \beta_{K}(Q) \ell(Q)+144 \delta|x-z| \\
& \leq 148 \delta|x-z|
\end{aligned}
$$

Lemma 4.1 is proved.

LEMMA 4.2. Suppose $\beta(K) \leq \delta, \quad \delta<\frac{1}{10}$ and $x, y, z$ satisfy

$$
\begin{aligned}
|x-z| & \geq \frac{1}{2} \max \{|x-y|,|y-z|\} \\
C^{-1} & \leq \frac{|x-y|}{|y-z|} \leq C,
\end{aligned}
$$


where $C$ is independent of $x, y, z$. Then

$$
\begin{aligned}
& \theta_{x}=|\arg (z-x)-\arg (y-x)| \leq C_{1} \delta \\
& \theta_{z}=|\arg (x-z)-\arg (y-z)| \leq C_{1} \delta
\end{aligned}
$$

where $C_{1}=19 \pi(1+C)$.

Proof. By Lemma 4.1,

$$
\operatorname{dist}(y, L) \leq 148 \delta|x-z|
$$

where $L$ is the straight line containing $[x, z]$. So, by our assumption,

$$
\sin \left(\theta_{x}\right)=\frac{\operatorname{dist}(y, L)}{|x-y|} \leq 148(1+C) \delta
$$

and this yields

$$
\theta_{x} \leq 19 \pi(1+C) \delta
$$

Similarly, we can also show

$$
\theta_{z} \leq 19 \pi(1+C) \delta
$$

Lemma 4.2 is proved.

LEMMA 4.3. Suppose $x, y, z$ satisfy

$$
|x-y|+|y-z| \leq(1+\varepsilon)|x-z|
$$

then

$$
\operatorname{dist}(y, L) \leq 2 \varepsilon^{\frac{1}{2}}|x-z|,
$$

where $L$ is a straight line containing $[x, z]$.

Proof. Let $L$ be a straight line containing $[x, z]$. By the Pythagorean theorem,

$$
2(\operatorname{dist}(y, L))^{2} \leq(|x-y|+|y-z|)^{2}-|x-z|^{2},
$$

and, then, Lemma 4.3 follows immediately, from our assumption. 
Lemma 4.4. Suppose $K_{1} \subset K$ and $K_{2} \subset \mathbb{C}$. Let $K^{\prime}=(K \backslash$ $\left.K_{1}\right) \cup K_{2}$. Then

$$
\beta_{K^{\prime}}(Q) \leq \frac{1}{\ell(Q)} \sup _{x \in K_{2} \cap 3 Q} \operatorname{dist}(x, K \cap 3 Q)+\beta_{K}(Q),
$$

where $Q$ is any dyadic square.

Proof. Without loss of generality we can assume that $K$ is compact. For any $x \in K_{2} \cap 3 Q$, where $Q$ is a dyadic square, there is $x_{Q} \in K \cup 3 Q$ such that $\left|x-x_{Q}\right|=\operatorname{dist}(x, K \cap 3 Q)$. Thus,

$$
\operatorname{dist}(x, L) \leq \operatorname{dist}(x, K \cap 3 Q)+\operatorname{dist}\left(x_{Q}, L\right),
$$

for any straight line $L$. By the definition of $\beta_{K}(Q)$, there is $L_{Q}$ such that

$$
\ell(Q) \beta_{K}(Q)=\inf _{L} \sup _{y \in K \cap 3 Q} \operatorname{dist}(y, L)=\sup _{y \in K \cap 3 Q} \operatorname{dist}\left(y, L_{Q}\right)
$$

and, then, using (4.2), we obtain

$$
\sup _{y \in K_{2} \cap 3 Q} \operatorname{dist}\left(y, L_{Q}\right) \leq \sup _{y \in K_{2} \cap 3 Q} \operatorname{dist}(y, K \cap 3 Q)+\ell(Q) \beta_{K}(Q) .
$$

Thus, if there exists $\left\{x_{j}\right\} \subset K_{2} \cap 3 Q$ such that

$$
\lim _{j \rightarrow \infty} \operatorname{dist}\left(x_{j}, L_{Q}\right)=\sup _{x \in K^{\prime} \cap 3 Q} \operatorname{dist}\left(x, L_{Q}\right),
$$

where $K^{\prime}=\left(K \backslash K_{1}\right) \cup K_{2}$, then, by (4.2),

$$
\ell(Q) \beta_{K^{\prime}}(Q) \leq \sup _{y \in K^{\prime} \cap 3 Q} \operatorname{dist}\left(y, L_{Q}\right)=\sup _{y \in K_{2} \cap 3 Q} \operatorname{dist}(y, K \cap 3 Q) .
$$

Hence, because of (4.3), Lemma 4.4 follows immediately. We assume now there is no $\left\{x_{j}\right\} \subset K_{2} \cap 3 Q$ such that (4.4) holds, which means that these exists $\left\{x_{j}\right\} \subset\left(K \backslash K_{1}\right) \cap 3 Q$ such that (4.4) holds. Thus, we have

$$
\begin{aligned}
\ell(Q) \beta_{K^{\prime}}(Q) & \leq \sup _{x \in K^{\prime} \cap 3 Q} \operatorname{dist}\left(y, L_{Q}\right) \\
& \leq \sup _{x \in K \cap 3 Q} \operatorname{dist}\left(y, L_{Q}\right)=\ell(Q) \beta_{K}(Q) .
\end{aligned}
$$


Lemma 4.4 now follows.

LEMMA 4.5. Suppose $\Gamma$ is a Jordan curve satisfying (4.0) with constant $\varepsilon<\frac{1}{100}$. Then $\Gamma$ is an $s$-quasicircle with constant $C_{0} \varepsilon$.

Proof. To show $\Gamma$ is a $s$-quasicircle with constant $C_{0} \varepsilon$, we need to verify that, for $x, z \in \Gamma$,

$$
|x-y|+|y-z| \leq\left(1+C_{0} \varepsilon\right)|x-z|, \quad \forall y \in \Gamma(x, z) .
$$

Without loss generality we assume $x=0, z>0$, and $|x-y| \leq$ $|y-z|$. Choose $y \in \Gamma(x, z)$. If $\Re(y) \geq 0$, by (4.0) and Lemma 4.1, it is easy to see that

$$
|x-y|+|y-z| \leq(1+148 \varepsilon)|x-z|
$$

We now assume $\Re(y)<0$. Lemma 4.5 follows immediately if we can prove:

Claim. If $y \in \Gamma(x, z)$ satisfies $\Re(y)<0$, then $|x-y| \leq 50 \varepsilon|x-z|$.

To prove this Claim, we observe that, if the Claim is false, i.e., there is $y_{0} \in \gamma(x, z)$ satisfying $\Re\left(y_{0}\right)<0$ such that $\left|x-y_{0}\right|>$ $50 \varepsilon|x-z|$, then, since $\Gamma$ satisfies (4.0) with constant $\varepsilon$, we have $\left|\arg \left(y_{0}\right)\right|>3 / 4 \pi$. We choose $Q_{0} \in \mathcal{D}$ containing $y_{0}$ and satisfying

$$
\left|x-y_{0}\right|<\ell\left(Q_{0}\right) \leq 2\left|x-y_{0}\right| \text {. }
$$

Then, $x \in 3 Q_{0}$, and $\Gamma\left(y_{0}, x\right)$ (and, thus, $\left.\Gamma\left(y_{0}, z\right) \cap 3 Q_{0}\right)$ is contained in a strip of width less than $2 \varepsilon \ell\left(Q_{0}\right)$.

Next, since $\Gamma$ is a Jordan curve, we can choose $Q_{1} \in \mathcal{D}$ such that $\ell\left(Q_{1}\right)<4 \varepsilon \ell\left(Q_{0}\right)$

$$
\operatorname{dist}\left(y_{0}, 3 Q_{1}\right)>0
$$

$Q_{1} \cap \Gamma\left(x, y_{0}\right) \neq \phi$ and $3 Q_{1} \cap \Gamma\left(y_{0}, z\right) \neq \phi$. By $(4.0), \quad \Gamma\left(y_{0}, x\right) \cap 3 Q_{1}$ and $\Gamma\left(y_{0}, z\right) \cap 3 Q_{1}$ are contained in a strip of width less than $2 \varepsilon \ell\left(Q_{1}\right)$ (see Figure 1). So, we can repeat the above process again. Recursively using (4.0) with constant $\varepsilon$ and the fact that $\Gamma$ is a Jordan curve, we can find $\left\{Q_{n}\right\} \subset \mathcal{D}$ such that 


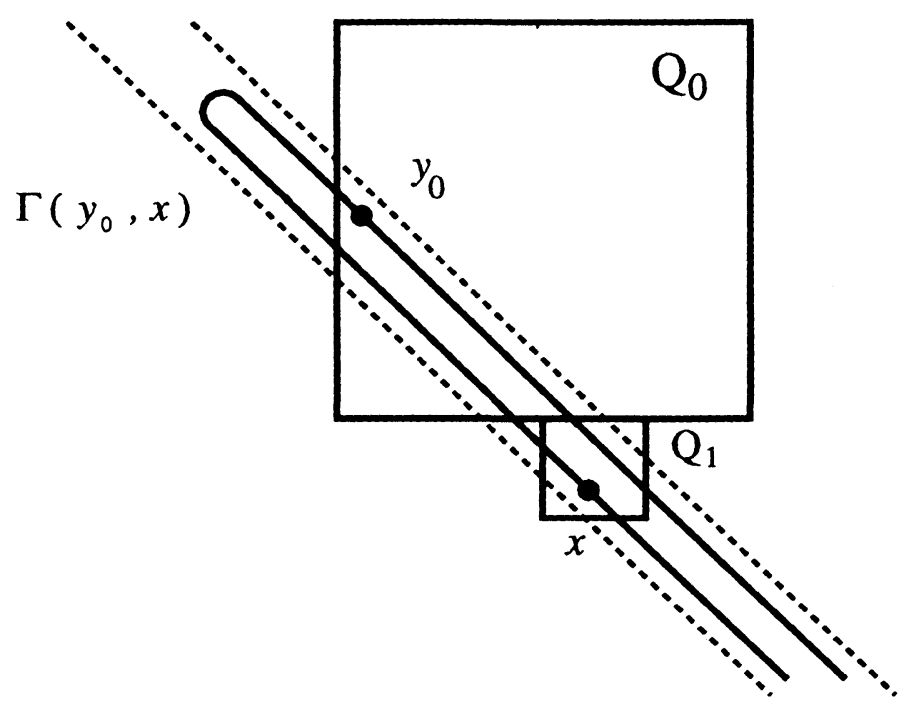

FIGURE 1.

(ii) $3 Q_{n+1} \subset 3 Q_{n}, \quad \forall n$,

(iii) $\ell\left(Q_{n+1}\right)<4 \varepsilon \ell\left(Q_{n}\right), \quad \forall n$,

(iv) $Q_{n} \cap \Gamma\left(x, y_{0}\right) \neq \phi$ and $Q_{n} \cap \Gamma\left(y_{0}, z\right) \neq \phi, \quad \forall n$.

Since $\varepsilon<\frac{1}{10}$, taking limits, we see that $\Gamma\left(x, y_{0}\right) \cap \Gamma\left(y_{0}, z\right) \neq\left\{y_{0}\right\}$, which contradicts the fact that $\Gamma$ is a Jordan curve. The Claim is, thus, proved. Therefore, Lemma 4.5 is proved.

Proof of Theorem 1.2. The strategy we use essentially follows [20]. Using Lemma 4.3, it is easy to show the converse part of Theorem 2.1. To prove the rest of Theorem 1.2, we choose $\mathcal{Z}_{n}=$ $\left\{z_{j}^{n}\right\} \subset K$ satisfying

$$
\begin{aligned}
& \left|z_{i}^{n}-z_{j}^{n}\right| \geq 2^{-n}, \quad i \neq j, \\
& K \subset \cup_{z_{j}^{n} \in \mathcal{Z}_{n}} D\left(z_{j}^{n}, 2^{-n}\right)
\end{aligned}
$$

and, also, let $\mathcal{K}_{n}=\mathcal{Z}_{n} \backslash \mathcal{Z}_{n-1}$. It is clear that $\mathcal{Z}_{n} \subset \mathcal{Z}_{n+1}$. and $\cup_{n} \mathcal{Z}_{n}$ is dense in $K$. If we can construct a Jordan curve containing 
$\cup_{n} \mathcal{Z}_{n}$, then, by continuity, the curve will contain the set $K$. To construct such a curve, by Lemma 4.5 , it is enough to construct $\Gamma_{n}$ containing $\mathcal{Z}_{n}$ with the uniform estimate: $\beta\left(\Gamma_{n}\right) \leq C_{0} \varepsilon, \forall n$.

Before we give the details of the construction of $\Gamma_{n}$, let us describe the idea of the construction. By (4.0), we can see that the points of $K$ are distributed in a strip-shaped region at any scale. So, at the fixed scale $2^{-n}$, because of (4.6), we may think of $\mathcal{Z}_{n}$ to consist of groups of points such that the points in the one group are close to each other, while points from different groups are sufficiently separated. Hence, we may use Construction-A (see below) to join the points in the same group, and use Construction-B (see below) to join different groups, and, by (4.0), essentially, we can control the estimate $\beta\left(\Gamma_{n}\right)$ uniformly.

Now, let us start the construction of $\Gamma_{n}$. Our construction is based on the following:

Construction-A $(x, y)$. Suppose $x, y \in \mathcal{Z}_{n}$ satisfy $|x-y| \leq 2^{-n+10}$. Without loss of generality we may assume $x=0$ and $y>0$. Taking all possible $x_{j} \in \mathcal{K}_{n+1} \cap D\left(x, \delta^{-1} 2^{-n-2}\right), j=1, \ldots, m$ which satisfy $\Re\left(x_{j}\right) \in[x, y]$ and $\Re\left(x_{j}\right)<\Re\left(x_{j+1}\right)$, we set

$$
\gamma_{[x, y]}=\cup_{j=0}^{m}\left[x_{j}, x_{j+1}\right]
$$

where $x_{0}=x, x_{m+1}=y$, and the construction is completed. (See Figure 2-(1). )

Construction- $\mathrm{B}\left(x_{1}, x_{2}, x_{3}\right)$. Suppose $x_{1}, x_{2}, x_{3} \in \mathbb{C}$ satisfy

$$
\left|x_{1}-x_{2}\right|<\left|x_{2}-x_{3}\right| \quad \text { and } \operatorname{dist}\left(x_{2}, L\right) \leq 2^{25} \delta\left|x_{1}-x_{3}\right| \text {, }
$$

and, up to a rigid transformation, $x_{1}=0, x_{3}>0$ and $\arg \left(x_{2}\right) \in$ $\left[0, \frac{3 \pi}{4}\right]$. Then,

- If $\arg \left(x_{2}\right) \leq \varepsilon$, we choose $z \in\left(x_{1}, x_{3}\right)$ such that $\left|x_{1}-x_{2}\right|=$ $\left|x_{2}-z\right|$, and set

$$
\gamma_{\left[x_{1}, x_{3}\right]}=\left[x_{1}, x_{2}\right] \cup\left[x_{2}, z\right] \cup\left[z, x_{3}\right] .
$$

- If $\arg \left(x_{2}\right)>\varepsilon$, we choose $z_{0}, z_{1}, \ldots, z_{s}$ such that $z_{0}=x_{1}, z_{1}=x_{2}$, and

$$
\arg \left(z_{j}\right)-\arg \left(z_{j+1}\right)=\varepsilon \quad \text { and } \quad \arg \left(z_{s-1}\right)-\arg \left(z_{s}\right)=\varepsilon_{0},
$$

$$
\left|z_{j}-z_{j+1}\right|=\left|z_{j}\right|, \quad j=0, \ldots, s-1,
$$


where $0<\varepsilon_{0} \leq \varepsilon, z_{s} \in\left(x_{1}, x_{3}\right)$. By (4.7), it is clear that

$$
\begin{aligned}
& \left|z_{j+1}\right|=\left|z_{j}\right| 2 \cos \varepsilon, \quad 0 \leq j \leq s-2, \\
& \left|z_{s}\right|=\left|z_{s-1}\right| 2 \cos \varepsilon_{0}, \quad \text { and } \\
& s \varepsilon+\varepsilon_{0}=\arg \left(x_{2}\right) .
\end{aligned}
$$

Let

$$
\gamma_{\left[x_{1}, x_{3}\right]}=\cup_{j=0}^{s}\left[z_{\jmath}, z_{j+1}\right], \quad z_{s+1}=x_{3} .
$$

The construction is completed. (See Figure 2-(2), $s=5$.)

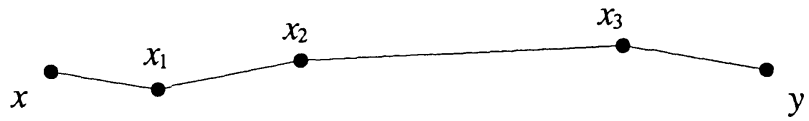

(1)

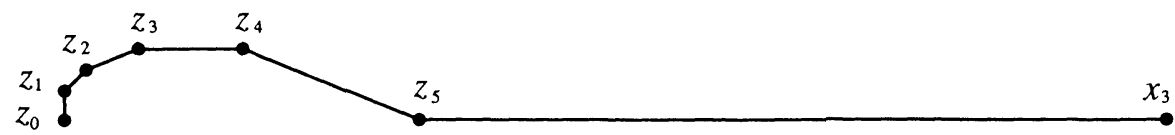

(2)

\section{Figure 2.}

We need the following lemma:

LEMMA 4.6. Suppose $\gamma$ is constructed by the above constructions. Then $\beta_{\gamma}(Q) \leq C_{0} \varepsilon$, for all $Q \in \mathcal{D}$. Moreover,

(i) If $\gamma$ is constructed by Construction- $A$, then

$$
\sup _{z \in \gamma} \operatorname{dist}(z,[x, y]) \leq 148 \delta|x-y|
$$

(ii) If $\gamma$ is constructed by Construction-B, i.e.

$$
\gamma_{\left[x_{1}, x_{3}\right]}=\cup_{\jmath=0}^{s}\left[z_{j}, z_{j+1}\right], \quad z_{0}=x_{1}, \quad z_{1}=x_{2} \quad \text { and } \quad z_{s+1}=x_{3} \text {, }
$$

then, let $\theta$ be the smaller angle between $\left[x_{1}, x_{2}\right]$ and $\left[x_{1}, z_{s}\right]$, $\sup \operatorname{dist}(z, L) \leq \varepsilon 2^{\theta \varepsilon^{-1}}\left|x_{1}-x_{2}\right| \quad$ and $\quad\left|x_{1}-z_{s}\right| \leq 2^{\theta \varepsilon^{-1}}\left|x_{1}-x_{2}\right|$. 
where $L$ is the straight line containing $\left[x_{1}, x_{3}\right]$.

Proof. Using (4.0), Lemma 4.1, Lemma 4.2 and the assumption of Construction-B, it is easy to prove $\beta_{\gamma} \leq C_{0} \varepsilon$. (i) follows from (4.0) and Lemma 4.1.

Let $\gamma=\cup_{j=0}^{m}\left[z_{j}, z_{j+1}\right], z_{0}=x_{1}, z_{1}=x_{2}$ and $z_{n+1}=x_{3}$. Without loss of generality we assume $x_{1}=0, x_{3}>0$. Note that

$$
\sup _{w \in \gamma} \operatorname{dist}(w, \mathbb{R})=\max _{0<j<s}\left|\Im\left(z_{j}\right)\right| \text {. }
$$

Also, by Construction-B, if $\arg \left(z_{j}\right)>2 \varepsilon$, then $\Im\left(z_{j+1}\right)>\Im\left(z_{j}\right)$. Thus, if we assume $z_{j_{0}}$ is maximal, $\arg \left(z_{j_{0}}\right) \leq 2 \varepsilon$. Hence, using (4.7), and (4.8),

$$
\begin{aligned}
\left|\Im\left(z_{j_{0}}\right)\right| & =\left|x_{1}-x_{2}\right|(2 \cos \varepsilon)^{j_{0}-1} \sin \left(\arg \left(z_{j_{0}}\right)\right) \\
& \leq\left|x_{1}-x_{2}\right| 2^{s-1} \varepsilon \\
& \leq \varepsilon 2^{\theta \varepsilon^{-1}}\left|x_{1}-x_{2}\right| .
\end{aligned}
$$

Here we used $\varepsilon(s-1) \leq \theta$. Similarly, we have

$$
\left|x_{1}-z_{s}\right| \leq\left|x_{1}-x_{2}\right| 2^{s-1} \leq 2^{\theta \varepsilon^{-1}}\left|x_{1}-x_{2}\right| \text {. }
$$

Thus, (ii) is proved.

We now use the induction on $n$ to construct the Jordan curve $\Gamma_{n}$ containing $\mathcal{Z}_{n}$ and satisfying the estimate: $\beta\left(\Gamma_{n}\right) \leq C_{0} \varepsilon$. The construction of $\Gamma_{1}$ is trivial. We omit it. We assume that we have constructed $\Gamma_{n}$ which satisfies the following conditions:

A-1. $\Gamma_{n}$ contains $\mathcal{Z}_{n}$ and consists of intervals such that the smaller angle between two adjacent intervals is at least $\pi-2 \varepsilon$. Also, $\Gamma_{n}=$ $\cup_{I} \gamma_{I}$, where $I=\left[z_{1}, z_{2}\right], z_{1}, z_{2} \in \mathcal{Z}_{n}, z_{1} \neq z_{2}$ such that $\gamma_{I} \cap \mathcal{Z}_{n}=$ $\left\{z_{1}, z_{2}\right\}$ and, if $\gamma_{I}$ is not an interval, i.e.,

$$
\gamma_{I}=\left[z_{1}, v_{1}\right] \cup\left[v_{1}, v_{2}\right] \cup \ldots \cup\left[v_{m}, z_{2}\right] .
$$

Then there exists $z \in \mathcal{Z}_{n}$ such that either $z_{1} \in\left(z, v_{1}\right)$, or, $v_{1} \in$ $\left(z, z_{1}\right)$, and

$$
\left|z_{1}-v_{1}\right| \geq \max \left\{2^{-n+2}, \frac{1}{2^{9}}\left|z-z_{1}\right|\right\}
$$


(See Figure $5, z_{1}=u_{m}$ ). Let $L_{n}=2^{\frac{3}{4} \pi \varepsilon^{-1}} 2^{-n-1}$, the next condition is

A-2. For $Q \in \mathcal{D}$, if $\ell(Q)>L_{n}$, then

$$
\beta_{\Gamma_{n}}(Q) \leq C_{0} \varepsilon \frac{L_{n}}{\ell(Q)}+\beta_{\Gamma_{n-1}}(Q)
$$

and, if $2^{-n} \leq \ell(Q) \leq L_{n}$, then either

$$
\beta_{\Gamma_{n}} \leq C_{0} \varepsilon \frac{2^{-n}}{\ell(Q)}+\beta_{\Gamma_{n-1}}(Q), \quad \text { or } \quad \beta_{\Gamma_{n}} \leq C_{0} \varepsilon
$$

and, finally, if $\ell(Q)<2^{-n}$, then

$$
\beta_{\Gamma_{n}}(Q) \leq C_{0} \varepsilon
$$

Construction of $\Gamma_{n+1}$. To construct $\Gamma_{n+1}$, we pick $w \in \mathcal{K}_{n+1}$ and choose $u_{0} \in \mathcal{Z}_{n}$ such that $\left|u_{0}-w\right|=\operatorname{dist}\left(w, \mathcal{Z}_{n}\right)$. and choose $u_{-1}, u_{1} \in \mathcal{Z}_{n}$ such that $u_{-1}$ is next to $u_{0}$ on the left side of $u_{0}$ on $\Gamma_{n}$, and $u_{1}$ is next to $u_{0}$ on the right side of $u_{0}$ on $\Gamma_{n}$.

Let $I=\left[u_{-1}, u_{0}\right], J=\left[u_{0}, u_{1}\right]$ and $\gamma_{I}, \gamma_{J} \subset \Gamma_{n}$. The construction around $w$ will be considered by several cases.

Case I. One of the intervals is of length less than $2^{-n+10}$.

Without loss of generality we assume $\ell(J)<2^{-n+10}$. Let us begin with the construction in the direction of the right side of $u_{0}$. Since $\left|u_{0}-u_{1}\right|<2^{-n+10}$, we use Construction-A $\left(u_{0}, u_{1}\right)$ to obtain a curve denoted by $P_{\left[u_{0}, u_{1}\right]}$ and, choose $u_{2} \in \mathcal{Z}_{n}$ at the right side of $u_{1}$ on $\Gamma_{n}$, and next to $u_{1}$. If $\left|u_{1}-u_{2}\right|<2^{-n+10}$, we repeat the above process to obtain $P_{\left[u_{1}, u_{2}\right]}$ and pick $u_{3} \in \mathcal{Z}_{n}$ such that $u_{3}$ is next to $u_{2}$ and at the right side of $u_{2}$ on $\Gamma_{n}$. We can keep this process going until, say, at $m$ th step,

$$
\left|u_{m}-u_{m+1}\right| \geq 2^{-n+10}
$$

We are now going to use Construction- $B$ to construct the curve $P_{\left[u_{m}, u_{m+1}\right]}$ and finish our construction in the direction of the right side of $w$. (See Figure 3, $\mathrm{m}=2$.)

It will be helpful for understanding our construction if we keep the following fact in mind: by (4.6) and (4.9), points in $\mathcal{K}_{n+1}$ that 


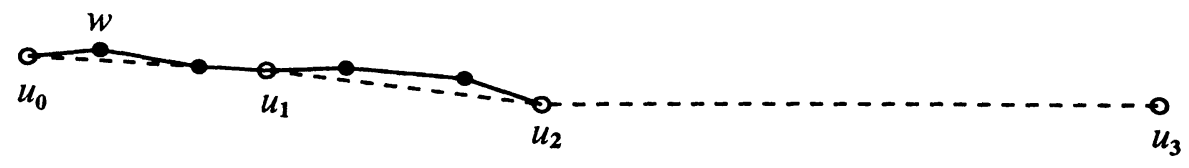

\section{Figure 3.}

have not been constructed yet, are either "close" to $u_{m}$, or "far away" from $u_{m}$. Let

$$
\left(D\left(u_{m}, 2^{-n}\right) \cap \mathcal{K}_{n+1}\right) \cup\left\{u_{m}\right\}=\left\{y_{j}\right\}_{j=1}^{s} .
$$

For convenience, we assume $u_{m-1}<0$, i.e., $\arg \left(u_{m-1}\right)=\pi, u_{m}=0$ and $\Re\left(y_{j}\right)<\Re\left(y_{j+1}\right)$, for $j=1, \ldots, s-1$.

Case I-1. $s>1$.

If $y_{s} \neq u_{m}$, we choose $v \in \mathbb{C}$ such that

$$
y_{s} \in\left(u_{m}, v\right) \quad \text { and } \quad\left|y_{s}-v\right|=2^{-n+1} .
$$

(See Figure 4-(1).) If $y_{s}=u_{m}$, we choose $v \in \mathbb{C}$ such that

$$
u_{m} \in\left(y_{s-1}, v\right) \quad \text { and } \quad\left|u_{m}-v\right|=2^{-n+1} .
$$

(See Figure 4-(2).) We are now going to verify the conditions permitting the use of Construction-B. The considerations are divided into two cases.

Case I-1.1. $\gamma_{\left[u_{m}, u_{m+1}\right]}=\left[u_{m}, u_{m+1}\right] . \quad$ (See Figure 4.)

We will perform Construction-B $\left(u_{m}, v, u_{m+1}\right)$. Since $y_{s}, u_{m}, u_{m+1}$ are in $K$, using Lemma 4.1 ,

$$
\operatorname{dist}\left(v,\left[u_{m}, u_{m+1}\right]\right) \leq 2 \operatorname{dist}\left(y_{s},\left[u_{m}, u_{m+1}\right]\right) \leq 296 \delta\left|u_{m}-u_{m+1}\right| .
$$

By (4.9), it is clear that

$$
\left|u_{m}-v\right|<\left|v-u_{m+1}\right|
$$

By (4.6), $2^{-n-1} \leq\left|u_{m}-y_{s}\right|<2^{-n}$. Note

$$
\left|u_{m-1}-u_{m}\right|<2^{-n+10} \quad \text { and } \quad u_{m-1}, u_{m}, y_{s} \in K
$$




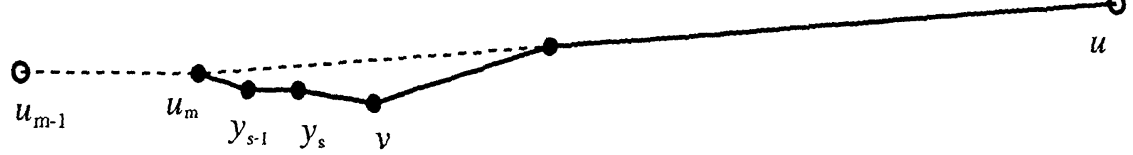

(1)

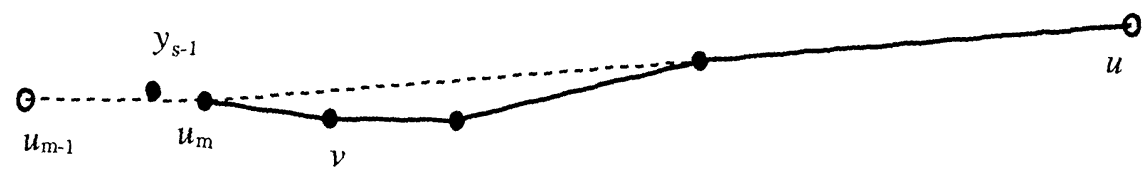

FiguRE 4.

and, by Lemma 4.2 and (4.0), it is easy to see $\left|\arg \left(y_{s}\right)\right|<\varepsilon$. Since $\arg (v)=\arg \left(y_{s}\right)$, and, by $\mathrm{A}-1$, the smaller angle between $\left[u_{m-1}, u_{m}\right]$ and $\left[u_{m}, u_{m+1}\right]$ is at least $\pi-2 \varepsilon$,

$$
\left|\arg (v)-\arg \left(u_{m+1}\right)\right|<3 \varepsilon
$$

Thus, we obtained the condition for Construction- $\mathrm{B}\left(u_{m}, v, u_{m+1}\right)$. Therefore, we apply Construction-B $\left(u_{m}, v, u_{m+1}\right)$ and obtain $P_{\left[u_{m}, u_{m+1}\right]}^{\prime}$. Let $y_{s_{0}}=u_{m}$ and let

$$
P_{\left[u_{m}, u_{m+1}\right]}=\left(P_{\left[u_{m}, u_{m+1}\right]}^{\prime} \backslash\left[u_{m}, y_{s}\right]\right) \cup\left(\cup_{j=s_{0}}^{s}\left[y_{j}, y_{j+1}\right]\right) .
$$

(See Figure 4.)

Case I-1.2. $\gamma_{\left[u_{m}, u_{m+1}\right]} \neq\left[u_{m}, u_{m+1}\right]$. (See Figure 5.)

We will perform Construction- $\mathrm{B}\left(u_{m}, v, v_{1}\right)$. Let

$$
\gamma_{\left\{u_{m}, u_{m+1}\right]}=\left[u_{m}, v_{1}\right] \cup \ldots \cup\left[v_{k}, u_{m+1}\right], \quad k \geq 1 .
$$

By induction A-1, there is $z \in \mathcal{Z}_{n}$ such that either $u_{m} \in\left(z, v_{1}\right)$ (see Figure 5-(1)) or, $v_{1} \in\left(u_{m}, z\right)$, (see Figure 5-(2)) and

$$
\left|u_{m}-v_{1}\right| \geq \max \left\{2^{-n+2}, \frac{1}{2^{9}}\left|u_{m}-z\right|\right\} .
$$



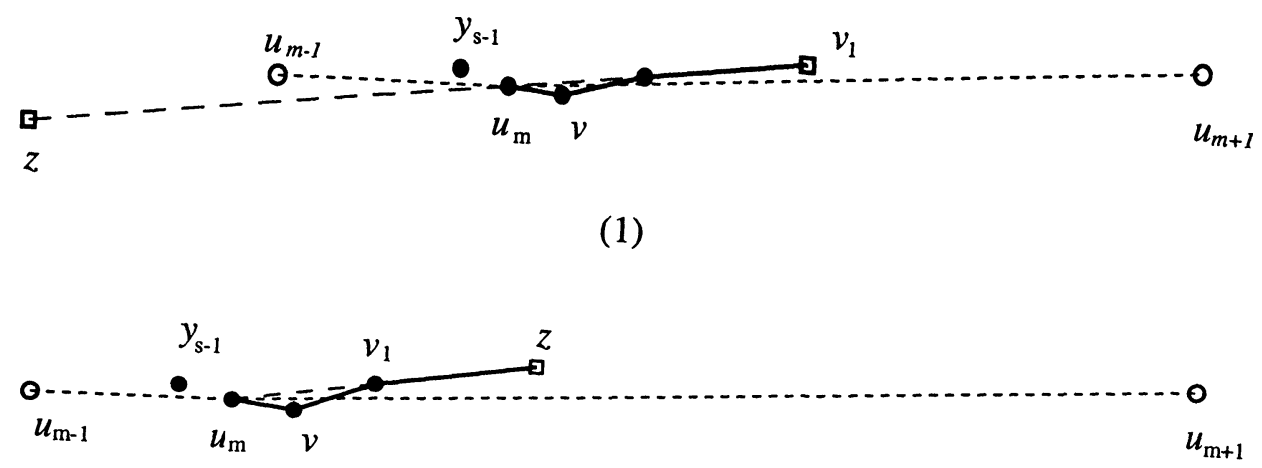

Figure 5. $\cdots$ the old curves

- the new curves.

Let us consider only in the case that $u_{m} \in\left(z, v_{1}\right)$, because the same argument works for the other case. To verify the conditions needed to perform Construction-B, we recall

$$
y_{s} \in D\left(u_{m}, 2^{-n}\right) \cap \mathcal{K}_{n+1} \quad \text { and } \quad z, u_{m} \in \mathcal{Z}_{n} .
$$

By (4.6), $\left|z-u_{m}\right|>\left|y_{s}-u_{m}\right|$ and, then, $\left|y_{s}-z\right|<2\left|z-u_{m}\right|$. Thus, if $L$ is the straight line containing $\left[u_{m}, z\right]$,

$$
\begin{aligned}
\operatorname{dist}(v, L) & \leq 4 \operatorname{dist}\left(y_{s}, L\right) & \\
& \leq 2^{10} \delta\left|u_{m}-z\right| & \text { by Lemma } 4.1 \\
& \leq 2^{19} \delta\left|u_{m}-v_{1}\right| & \text { by }(4.11) .
\end{aligned}
$$

By the choice of $v$ and (4.11), it is clear that

$$
\left|u_{m}-v\right|<\left|u_{m}-v_{1}\right|
$$

Using the same argument as in Case I-1.1, we can prove

$$
\left|\arg (v)-\arg \left(v_{1}\right)\right|<3 \varepsilon .
$$

We can, therefore, use Construction- $\mathrm{B}\left(u_{m}, v, v_{1}\right)$ and obtain a curve $P_{\left[u_{m}, u_{m+1}\right]}^{\prime}$. Let

$$
P_{\left[u_{m}, u_{m+1}\right]}=\left(P_{\left[u_{m}, u_{m+1}\right]}^{\prime} \backslash\left[u_{m}, y_{s}\right]\right) \cup\left(\cup_{j=s_{0}}^{s-1}\left[y_{j}, y_{j+1}\right]\right)
$$


where $y_{s_{0}}=u_{m}$.

Case I-2. $s=1$.

If there is no $x \in \mathcal{K}_{n+1}$ such that $\Re(x) \in\left(u_{m-1}, u_{m}\right)$, we let

$$
P_{\left[u_{m}, u_{m+1}\right]}=\gamma_{\left[u_{m}, u_{m+1}\right]}
$$

and, otherwise, we choose a point $x \in \mathcal{K}_{n+1}, \Re(x) \in\left[u_{m-1}, u_{m}\right)$ such that $x$ is nearest to $u_{m}$. We may only consider the case $\gamma_{\left[u_{m}, u_{m+1}\right]} \neq$ $\left[u_{m}, u_{m+1}\right]$, because the same argument works for the other case. Let $\theta$ be the smaller angle between $\left[u_{m}, v_{1}\right]$ and $\left[u_{m}, x\right]$, where $v_{1}$ is as in Case I-1.2. We note that, if $\theta>\pi-2 \varepsilon$, we are done by letting

$$
P_{\left[u_{m}, u_{m+1}\right]}=\gamma_{\left[u_{m}, u_{m+1}\right]} .
$$

Let us now assume that $\theta \leq \pi-2 \varepsilon$, i.e.,

$$
\left|\arg (x)-\arg \left(v_{1}\right)\right| \leq \pi-2 \varepsilon
$$

We choose $v \in \mathbb{C}$ such that $u_{m} \in(x, v)$ and $\left|u_{m}-v\right|=2^{-n+1}$. (See Figure 6.)

Since $x \neq u_{m-1}$, by (4.6), $2^{-n-1} \leq\left|x-u_{m-1}\right|<2^{-n}$, and $\left|u_{m-1}-u_{m}\right|<2^{-n+10}$, then, by Lemma $4.2, \quad\left|\arg (x)-\arg \left(u_{m-1}\right)\right|<$ $\varepsilon$. Hence, by A-1 and $|\arg (v)-\arg (x)|=\pi$,

$$
\left|\arg (v)-\arg \left(v_{1}\right)\right|<3 \varepsilon \text {. }
$$

Using the same argument as we did in Case I-1.2, we can prove $\operatorname{dist}(v, L) \leq 2^{9} \operatorname{dist}(x, L) \leq 2^{26} \delta\left|u_{m}-v_{1}\right|, \quad$ and $\quad\left|u_{m}-v\right|<\left|v-v_{1}\right|$ where $L$ is a straight line containing $\left[u_{m}, v_{1}\right]$. The condition for Construction-B is, thus, verified. Hence, we apply Construction$\mathrm{B}\left(u_{m}, v, v_{1}\right)$ and obtain $P_{\left[u_{m}, v_{1}\right]}^{\prime}$. Let

$$
P_{\left[u_{m}, u_{m+1}\right]}=P_{\left[u_{m}, v_{1}\right]}^{\prime} \cup\left(\cup_{j=1}^{k}\left[v_{j}, v_{j+1}\right]\right) .
$$

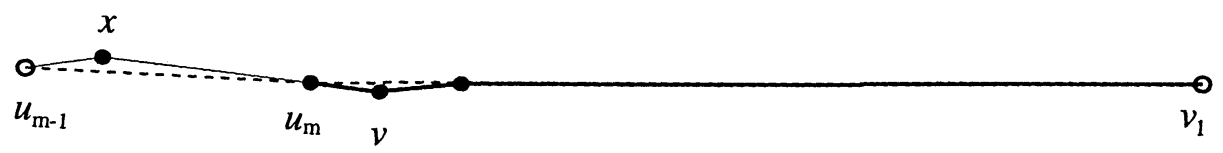

Figure 6. 
Here, we assume

$$
\gamma_{\left[u_{m}, u_{m+1}\right]}=\cup_{j=0}^{k}\left[v_{j}, v_{j+1}\right]
$$

where $v_{0}=u_{m}$ and $v_{k+1}=u_{m+1}$. Similarly, we can construct $P_{\left[u_{m}, u_{m+1}\right]}$ when $\gamma_{\left[u_{m}, u_{m+1}\right]}=\left[u_{m}, u_{m+1}\right]$. We omit the details. Let

$$
S_{\left[u_{0}, u_{m+1}\right]}=\cup_{j=0}^{m} P_{\left[u_{j}, u_{j+1}\right]} .
$$

We thus complete the construction for the right side of $u_{0}$. The construction in the other direction is exactly same and we omit it. Assume we end up at the $\left(m^{\prime}-1\right)$ th step. Let

$$
S_{\left[u_{-m^{\prime}}, u_{0}\right]}=\cup_{j=0}^{m^{\prime}-1} P_{\left[u_{-j}, u_{-j-1}\right]} .
$$

We set

$$
\gamma_{n e w, 1}=S_{\left[u_{-m^{\prime}}, u_{0}\right]} \cup S_{\left[u_{0}, u_{m+1}\right]}, \quad \text { and } \quad \gamma_{o l d, 1}=\Gamma_{n}\left(u_{-m^{\prime}} u_{m+1}\right) .
$$

We therefore completed the construction in Case I.

Case II. The length of both $I$ and $J$ are greater or equal to $2^{-n+10}$. Let

$$
\begin{aligned}
& \gamma_{\left[u_{0}, u_{1}\right]}=\left[u_{0}, v_{1}\right] \cup \ldots \cup\left[v_{m}, u_{1}\right], \\
& \quad \text { and } \\
& \gamma_{\left[u_{0}, u_{-1}\right]}=\left[u_{0}, x_{1}\right] \cup \ldots \cup\left[x_{m^{\prime}}, u_{-1}\right] .
\end{aligned}
$$

Without loss of generality we can assume $u_{0}=0, v_{1}>0$ and $0<$ $\arg (w)<\frac{\pi}{2}+\varepsilon$. Indeed, if $\arg (w) \geq \frac{\pi}{2}+\varepsilon$, then by $\mathrm{A}-1$, since the smaller angle between $\left[x_{1}, u_{0}\right]$ and $\left[u_{0}, v_{1}\right]$ is larger than $\pi-2 \varepsilon$, $\left|\arg (w)-\arg \left(x_{1}\right)\right|<\frac{\pi}{2}+\varepsilon$, and, so, we may exchange $u_{-1}$ and $u_{1}$, and thus, exchange $x_{1}$ and $v_{1}$. Let

$$
\left(D\left(u_{0}, 2^{-n}\right) \cap \mathcal{K}_{n+1}\right) \cup\left\{u_{0}\right\}=\left\{y_{j}\right\}_{j=1}^{s} .
$$

It is clear that $w \in\left\{y_{j}\right\}_{j=1}^{s}$ and $s>1$. We assume the index $j$ is chosen so that

$$
\Re\left(y_{j} e^{-i \arg (w)}\right)<\Re\left(y_{j+1} e^{-i \arg (w)}\right), \forall j .
$$

Since $w \in\left\{y_{j}\right\}_{j=1}^{s}$,

$$
\Re\left(y_{1} e^{-i \arg (w)}\right) \leq 0<\Re\left(w e^{-i \arg (w)}\right) \leq \Re\left(y_{s} e^{-i \arg (w)}\right) .
$$


Choose $w_{1}$ such that $y_{s} \in\left(u_{0}, w_{1}\right)$ and $\left|y_{s}-w_{1}\right|=2^{-n+1}$. Choose $w_{2}$ such that if $y_{1} \neq u_{0}, y_{1} \in\left(w_{2}, u_{0}\right)$, otherwise, $y_{1} \in\left(w_{2}, y_{2}\right)$ and $\left|w_{2}-y_{1}\right|=2^{-n+1}$. We will use Construction- $\mathrm{B}\left(u_{0}, w_{1}, v_{1}\right)$ and Construction- $\mathrm{B}\left(u_{0}, w_{2}, x_{1}\right)$ to construct the curve.

Using the same method as we used in Case I-1, we can show

$$
\operatorname{dist}\left(w_{1}, L\right) \leq 2^{19} \delta\left|u_{0}-v_{1}\right|
$$

where $L$ is the straight line containing $\left[u_{0}, v_{1}\right]$ and it is easy to see $\left|u_{0}-w_{1}\right|<\left|u_{0}-v_{1}\right|$. Thus, applying Construction-B $\left(u_{0}, v, v_{1}\right)$, we obtain $P_{\left[u_{0}, u_{1}\right]}^{\prime}$. Let

$$
P_{\left[u_{0}, u_{1}\right]}=\left(P_{\left[u_{0}, u_{1}\right]}^{\prime} \backslash\left[u_{0}, y_{s}\right]\right) \cup\left(\cup_{j=s_{0}}^{s}\left[y_{j}, y_{j+1}\right]\right)
$$

where $y_{s_{0}}=u_{0}$.

The same argument can be used to construct the curve $P_{\left[u_{-1}, u_{0}\right]}$ between $u_{-1}$ and $u_{0}$, we omit the details. (See Figure 7.) Let

$$
\gamma_{n e w, 1}=P_{\left[u_{-1}, u_{0}\right]} \cup P_{\left[u_{0}, u_{1}\right]} \quad \text { and } \quad \gamma_{o l d, 1}=\Gamma_{n}\left(u_{-1}, u_{1}\right)
$$

Therefore, the construction in Case II is finished.

Let

$$
\Gamma_{n+1,1}=\left(\Gamma_{n} \backslash \gamma_{o l d, 1}\right) \cup \gamma_{n e w, 1}
$$

REMARK 1 . The construction is always finished by Construction$\mathrm{B}\left(x_{1}, x_{2}, x_{3}\right)$. Let $\left[y_{s}, x_{3}\right]$ be the last interval in Construction- $\mathrm{B}$. We then have

$$
\left|y_{s}-x_{3}\right| \geq \frac{3}{4}\left|x_{1}-x_{3}\right|
$$

Indeed, by Lemma 4.6 , if $\theta \leq 3 \varepsilon$, by our choice of $x_{2},\left|x_{1}-x_{2}\right| \leq$

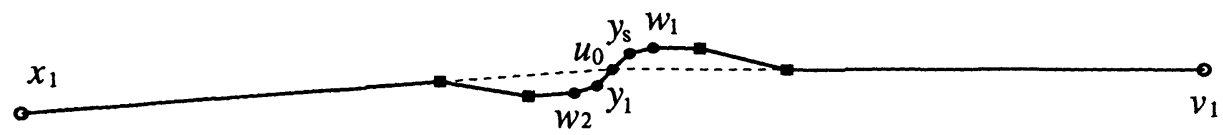

Figure 7. 
$2^{-9}\left|x_{1}-x_{3}\right|$, then

$$
\begin{aligned}
\left|y_{s}-x_{3}\right| & =\left|x_{1}-x_{3}\right|-\left|x_{1}-y_{s}\right| \\
& \geq\left|x_{1}-x_{3}\right|-2^{\theta \varepsilon^{-1}}\left|x_{1}-x_{2}\right| \\
& \geq\left(1-2^{-6}\right)\left|x_{1}-x_{3}\right| \\
& \geq \frac{3}{4}\left|x_{1}-x_{3}\right|
\end{aligned}
$$

if $\theta>3 \varepsilon$, (which happens only in Case II) then, by (4.14), we have

$$
\operatorname{dist}\left(x_{2}, L\right) \leq 2^{19} \delta\left|x_{1}-x_{3}\right| \text {. }
$$

Then, since $\theta<\frac{3}{4} \pi$,

$$
\left|x_{1}-x_{2}\right| \leq \frac{\operatorname{dist}\left(x_{2}, L\right)}{\sin (3 \varepsilon)} \leq 2^{19} \varepsilon^{-1} \delta\left|x_{1}-x_{3}\right|
$$

Thus,

$$
\begin{aligned}
\left|y_{s}-x_{3}\right| & \geq\left|x_{1}-x_{3}\right|-2^{\theta \varepsilon^{-1}}\left|x_{1}-x_{2}\right| \\
& \geq\left|x_{1}-x_{3}\right|-2^{\frac{3}{4} \pi \varepsilon^{-1}}\left|x_{1}-x_{2}\right| \\
& \geq\left(1-\delta^{\frac{1}{4}} 2^{18}\right)\left|x_{1}-x_{3}\right| \\
& \geq \frac{3}{4}\left|x_{1}-x_{3}\right| .
\end{aligned}
$$

Remark 2. In Case I, by (4.0), (4.10)-(4.13) and Lemma 4.6, it is easy to prove

$$
\sup _{z \in \gamma_{\text {new }, 1}} \operatorname{dist}\left(z, \gamma_{\text {old }, 1}\right) \leq C_{0} \varepsilon 2^{-(n+1)}
$$

for a numerical constant $C_{0}$. In Case II, we have more to say. Let us use the same notations as before. Recall $\arg \left(w_{1}\right)=\arg \left(y_{s}\right)$ and $\arg \left(w_{2}\right)=\arg \left(y_{1}\right)$, or, $\arg \left(y_{2}\right)$. If $\arg \left(w_{1}\right) \leq 4 \varepsilon, \mid \arg \left(w_{2}\right)-$ $\arg \left(x_{1}\right) \mid \leq 7 \varepsilon$. Here, we used (4.0) and A-1. Hence, by Lemma 4.6, (4.16) is also true. If $\arg \left(w_{1}\right)>4 \varepsilon,\left|\arg \left(w_{2}\right)-\arg \left(x_{1}\right)\right|>\varepsilon$. Here, we also used (4.0) and A-1. Since $\arg \left(w_{1}\right)<\frac{\pi}{2}+\varepsilon$,

$$
\left|\arg \left(w_{2}\right)-\arg \left(x_{1}\right)\right| \leq \frac{\pi}{2}+4 \varepsilon<\frac{3}{4} \pi
$$


Thus, by (4.14),

$$
\begin{aligned}
& \left|u_{0}-w_{1}\right| \leq 2^{19} \varepsilon^{-1} \delta\left|u_{0}-v_{1}\right|, \\
& \left|u_{0}-w_{2}\right| \leq 2^{19} \varepsilon^{-1} \delta\left|u_{0}-x_{1}\right| .
\end{aligned}
$$

and, let $L_{n+1}=2^{\frac{3}{4} \pi \varepsilon^{-1}} 2^{-n}$, then

$$
L_{n+1} \leq \frac{1}{4} \min \left\{\left|u_{0}-v_{1}\right|,\left|u_{0}-x_{1}\right|\right\} .
$$

Therefore, using Lemma 4.6, it is easy to prove that, for $Q \in \mathcal{D}$, $\ell(Q)<L_{n+1}$,

$$
\beta_{\gamma_{n e w, 1}}(Q) \leq C_{0} \varepsilon
$$

To continue our construction of $\Gamma_{n+1}$, we pick $w \in \mathcal{K}_{n+1} \backslash \Gamma_{n+1,1}$. Repeat the above construction, we obtain $\gamma_{n e w, 2}$ and $\gamma_{o l d, 2}$. Let

$$
\Gamma_{n+1,2}=\left(\Gamma_{n} \backslash\left(\gamma_{\text {old }, 1} \cup \gamma_{\text {old }, 2}\right)\right) \bigcup\left(\gamma_{n e w, 1} \cup \gamma_{\text {new }, 2}\right) \text {. }
$$

In general, at the kth step, we obtain

$$
\Gamma_{n+1, k}=\left(\Gamma_{n} \backslash \cup_{j=1}^{k} \gamma_{o l d, j}\right) \cup\left(\cup_{j=1}^{k} \gamma_{n e w, j}\right)
$$

and, we keep doing this until $\mathcal{K}_{n+1}$ is exhausted. We obtain $\left\{\gamma_{n e w, j}\right\}$ and $\left\{\gamma_{o l d, j}\right\}$. Let

$$
\gamma_{\text {new }}=\cup\left\{\gamma_{\text {new }, j}\right\} \quad \text { and } \quad \gamma_{\text {old }}=\bigcup\left\{\gamma_{\text {old }, j}\right\}
$$

and let

$$
\Gamma_{n+1}=\left(\Gamma_{n} \backslash \gamma_{\text {old }}\right) \cup \gamma_{\text {new }}
$$

It is clear that $\Gamma_{n+1}=\Gamma_{n+1, k_{0}}$, for some $k_{0}$, since $\mathcal{K}_{n+1}$ is finite, and $\mathcal{Z}_{n+1} \subset \Gamma_{n+1}$. We now verify A-1 and A-2 for $\Gamma_{n+1}$. By our construction, it is easy to see A-1 is true for $\Gamma_{n+1}$. We need to check A-2. Choose any $Q \in \mathcal{D}$. If $\ell(Q)<2^{-(n+1)}$, by $(4.6)$, it is easy to prove

$$
\beta_{\Gamma_{n+1}}(Q) \leq C_{0} \varepsilon
$$

which is A-2-(iii). By Lemma 4.4,

$$
\beta_{\Gamma_{n+1}}(Q) \leq \frac{1}{\ell(Q)} \sup _{z \in \gamma_{n e w} \cap 3 Q} \operatorname{dist}\left(z, \gamma_{o l d}\right)+\beta_{\Gamma_{n}}(Q)
$$


By Remark 1, (4.15), it is easy to see that the sets $\left\{\gamma_{n e w, k} \backslash \gamma_{o l d, k}\right\}$ are separated sufficiently far away from each other so that there exists $k_{1}$ such that

$$
\sup _{z \in \gamma_{\text {new }} \cap 3 Q} \operatorname{dist}\left(z, \gamma_{\text {old }}\right)=\sup _{z \in \gamma_{\text {new }, k_{1}} \cap 3 Q} \operatorname{dist}\left(z, \gamma_{\text {old }, k_{1}}\right)
$$

Without loss of generality we may assume $k_{1}=1$ and $3 Q \cap\left(\gamma_{n e w, 1} \backslash \gamma_{\text {old }, 1}\right) \neq \phi$. So, for $\ell(Q) \geq 2^{-(n+1)}$, if $\gamma_{n e w, 1}$ is constructed by Case I, by (4.16) and (4.19)-(4.20),

$$
\beta_{\Gamma_{n+1}}(Q) \leq C_{0} \varepsilon \frac{2^{-(n+1)}}{\ell(Q)}+\beta_{\Gamma_{n}}(Q),
$$

which implies A-2(i)-(ii), and, if $\gamma_{\text {new,1 }}$ is constructed by Case II, then either (4.16) holds which yields (4.21), or, (4.18) holds if $2^{-(n+1)}<\ell(Q) \leq L_{n+1}$. But, (4.17) implies $\Gamma_{n+1} \cap 3 Q=\gamma_{n e w, 1} \cap 3 Q$, thus, by (4.18), we obtain

$$
\beta_{\Gamma_{n+1}}(Q) \leq C_{0} \varepsilon
$$

So, A-2-(ii) is verified for Case II. Finally, we need to verify A2-(i) when $\gamma_{n e w, 1}$ is constructed by Case II. Since $\gamma_{n e w, 1}$ is built by Construction-B, using Lemma 4.6-(ii), it is easy to obtain

$$
\sup _{z \in \gamma_{\text {new }, 1} \cap 3 Q} \operatorname{dist}\left(z, \gamma_{o l d, 1}\right) \leq 2^{4} \varepsilon L_{n+1},
$$

and, then, by (4.19)-(4.20), A-2-(i) is verified. Therefore, we completed the construction of the desired $\Gamma_{n+1}$.

Let $n$ tend to infinity, we obtain a Jordan curve $\Gamma$ which contains $K$. For $Q \in \mathcal{D}$, let $\ell(Q)=2^{-s}, s \geq 0$, we choose $n_{0}$ large enough such that, for any $n>n_{0}, \ell(Q)>L_{n}$. Recall $L_{n}=2^{\frac{3}{4} \pi \varepsilon^{-1}} 2^{-n-1}$. We choose $m$ such that

$$
L_{m}<\ell(Q) \leq L_{m-1}
$$

Since $2 L_{k}=L_{k-1}$

$$
L_{n}+L_{n-1}+\ldots+L_{m}=L_{m}\left(2^{-(n-m)}+\ldots+1\right) \leq 2 \ell(Q) .
$$


Thus, using A-2-(i) repeatedly,

$$
\begin{aligned}
\beta_{\Gamma_{n}}(Q) & \leq C_{0} \varepsilon \frac{1}{\ell(Q)}\left(L_{n}+L_{n-1}+\ldots+L_{m}\right)+\beta_{\Gamma_{m-1}}(Q) \\
& \leq 2 C_{0} \varepsilon+\beta_{\Gamma_{m-1}}(Q) .
\end{aligned}
$$

Now, we need A-2-(ii). Let $t<m$ be the index such that $\Gamma_{t}$ is the first one such that

$$
\beta_{\Gamma_{t}} \leq C_{0} \varepsilon
$$

By A-2-(ii) and (iii), it is clear that $t \geq s$. Using A-2-(ii) recursively,

$$
\begin{aligned}
\beta_{\Gamma_{m-1}}(Q) & \leq C_{0} \varepsilon \frac{1}{\ell(Q)}\left(2^{-m+1}+\ldots+2^{-(t+1)}\right)+\beta_{\Gamma_{t}}(Q) \\
& \leq 3 C_{0} \varepsilon
\end{aligned}
$$

We therefore obtain

$$
\beta_{\Gamma_{n}}(Q) \leq 5 C_{0} \varepsilon, \quad \forall n>n_{0}
$$

Since the estimate is independent of $n$,

$$
\beta_{\Gamma}(Q) \leq 5 C_{0} \varepsilon
$$

Let $Q_{0}=[0,1] \times[0,1]$. It is easy to see that $\Gamma$ we just constructed is contained in $Q_{0}$. Then, for $Q \in \mathcal{D}, \ell(Q)=2^{-s}$ with $s<0$, by (4.22),

$$
\beta_{\Gamma}(Q) \leq \beta_{\Gamma}\left(Q_{0}\right) \leq 5 C_{0} \varepsilon
$$

Therefore, Theorem 1.2 is proved.

REMARK 3. The curve $\Gamma$ we constructed here does not cross infinity. However, using our construction, it is easy to extend $\Gamma$ to be the Jordan curve $\Gamma^{\prime}$ which crosses infinity and satisfies $\beta_{\Gamma^{\prime}}(Q) \leq$ $C_{0} \varepsilon$, and therefore, which is an image of $\mathbb{R}$ under a quasiconformal mapping. We leave this to reader. 
Acknowledgements. I would like to thank Professor P. W. Jones, for his encouragement and for being so generous with both his time and ideas. Next, I would like to thank Professor R. R. Coifman for his very helpful suggestions. I am also grateful to Professors M. Christ, G. David, T. Murai and S. Semmes for their valuable comments. I also thank Professor Guido Weiss for reading this paper and correcting my mistakes in English.

\section{REFERENCES}

[1] L.V. Ahlfors, Lectures on Quasiconformal Mappings, Van Nostrand, Princeton. N.J., 1966, Theorem IV.5.

[2] A.S. Besicovitch, On the fundamental geometrical properties of linearly measurable plane sets of points, Math. Ann., 116 (1939), 349-357.

[3] A.P. Calderón, Cauchy integrals on Lipschitz curves and related topics, Proc. Acad. Sci. U.S.A., (1977) 1324-1327.

[4] A.P. Calderón, Commutators, singular integrals on Lipschitz curves and applications, ICM Helsinki, (1978).

[5] M. Christ, Lectures on Singular Integral Operators, Regional Conference Series in Math., 77, Amer. Math. Soc., 1990.

[6] M. Christ, $A T(b)$ theorem with remarks on analytic capacity and the Cauchy integral, Colloq. Math., LX/LXI (1990).

[7] R. R. Coifman and C. Fefferman, Weighted norm inequalities for maximal functions and singular integrals, Studia Math., 51 (1974), 241-250.

[8] R. R. Coifman, A. McIntosh and Y. Meyer, L'integral de Cauchy definit im opérateor borne sur $L^{2}$ pour les courbes lipschitziennes, Ann. of Math., 116 (1982), 361-387.

[9] R. R. Coifman and G. Weiss, Hardy space and their uses in analysis, Bulletin Amer. Math. Soc., 83 (1977), 569-645.

[10] G. David, Opératurs integraux singuliers sur certaines courbes du plan complex, Ann. Sci. École. Norm. Sup., 17 (1984), 157-189.

[11] G. David and S. Semmes, Singular intgrals and rectifiable sets in $R^{n}$ : Au-delá des graphs lipschitziens, Astérisque, 193 (1991).

[12] A.M. Davie and B. Øksendal, Analytic capacity and differentiability properties of finely harmonic functions, Acta Math., 149 (1982).

[13] K. J. Falconer, The Geometry of Fractal Sets, Cambridge University Press, Cambridge, 1985.

[14] X. Fang, The Cauchy integral of Calderon and analytic capacity, dissertation, Math. Dept., Yale, 1990. 
[15] J. Garnett, Positive length but zero analytic capacity, Proc. Amer. Math. Soc., 24 (1970), 696-699.

[16] J. Garnett, Analytic Capacity and Measure, Lecture Notes in Math. 297, Springer Verlag, 1972.

[17] D. Jerison and C. Kenig, Hardy spaces, $A_{\infty}$, and singular integrals on chord-arc domains, Math. Scand., 50 (1982), 221-247.

[18] D. Jerison and C. Kenig, Boundary behavior of harmonic functions in non-tangentially accessible domains, Advances in Math., 46 (1982), 80147.

[19] P. W. Jones, Square Functions, Cauchy Integrals, Analytic Capacity and Harmonic Measure, Harmonic analysis and partial differential equations, Springer Lectures in Math. No. 1384 (1987), 24-68.

[20] P. W. Jones, Rectifiable sets and the traveling salesman problem, Invent. Math., 102 (1990), 1-15.

[21] P. W. Jones and D. E. Marshall, Critical points of Green's function, harmonic measure and the corona problem, Ark. Math., 23 (1985), 281314.

[22] P. W. Jones and T. Murai, Positive analytic capacity but zero Buffon needle probability, Pacific J. Math., 133 (1988), 99-114.

[23] J.-L. Journé, Calderón-Zygmund Operators, Pseudo-Differential Operators, and the Cauchy Integral of Calderón, Lecture Notes in Math., 994, Springer-Verlag, New York, 1983.

[24] D. E. Marshall, Removable Sets for Bounded Analytic Functions, in Linear and Complex Analysis - 199 Research Problems, Lecture Notes in Math., 1043 (1984).

[25] P. Mattila, A class of sets with positive length and zero analytic capacity, Ann. Acad. Sci. Fenn. Ser. AI, 10 (1985), 387-395.

[26] T. Murai, Construction of $H^{1}$ functions concerning the estimate of analytic capacity, Bull. London Math., 19 (1986), 154-160.

[27] T. Murai A Real Variable Method for the Cauchy Transform, and Analytic Capacity, Lecture Notes in Math., 1307, Springer-Verlag, New York, 1988.

[28] E. M. Stein, Singular Integrals and Differentiability Properties of Functions, Princeton Univ. Press, Princeton, 1970.

[29] L. Zalcman, Analytic Capacity and Rational Approximation, Lecture Notes in Math., 50, Springer-Verlag, Berlin, 1968. 
Received August 10, 1992, revised March 1, 1993 and accepted for publication March 16, 1993.

\section{WASHINGTON UNIVERSITY}

ST. LOUIS, MisSOURI 63130-4899

E-mail address: fang@math.wustl.edu 



\section{PACIFIC JOURNAL OF MATHEMATICS}

Volume $166 \quad$ No. $2 \quad$ December 1994

Geometric aspects of Bäcklund transformations of Weingarten

submanifolds

STEVEN BUYSKE

Multipliers between invariant subspaces of the backward shift

225

ROBERT BRUCE CROFOOT

The Cauchy integral, analytic capacity and subsets of quasicircles

XIANG FANG

The number of lattice points within a contour and visible from the origin 295

Douglas Austin Hensley

On flatness of the Coxeter graph $E_{8}$

305

MASAKI IZUMI

Immersions up to joint-bordism

GUI SONG LI

Generalization of the Hilbert metric to the space of positive definite matrices

CARlangelo Liverani and Maciej WojtKowski

Periodicity, genera and Alexander polynomials of knots

SWATEE NAIK

On divisors of sums of integers. $\mathrm{V}$

ANDRÁS SÁRKÖZY and CAMERON LEIGH STEWART

Approximately inner automorphisms on inclusions of type $\mathrm{III}_{\lambda}$-factors

CARL WINSLøW

Correction to: "A convexity theorem for semisimple symmetric spaces"

KARL-HERMANN NEEB

Correction to: "Periodic points on nilmanifolds and solvmanifolds"

EDWARD KEPPELMANN

Correction to: "Partially measurable sets in measure spaces" 